\title{
Multiphysics modeling and simulation of high-solids dilute-acid pretreatment of corn stover in a steam-explosion reactor
}

\author{
Hariswaran Sitaraman ${ }^{\mathrm{a}}$, Erik M. Kuhn ${ }^{\mathrm{b}}$, Ambarish Naga ${ }^{\mathrm{a}}$, Michael A. \\ Sprague $^{\mathrm{a}}$, Melvin P. Tucker ${ }^{\mathrm{b}}$, Jonathan J. Stickel ${ }^{\mathrm{b}, *}$ \\ ${ }^{a}$ Computational Science Center \\ ${ }^{b}$ National Bioenergy Center, National Renewable Energy Laboratory, 15013 Denver West \\ Pkwy, Golden, CO, 80401
}

\begin{abstract}
Pretreatment of lignocellulosic biomass, by which the accessibility of cellulose to enzymes is enhanced, is an important step in the biochemical conversion of biomass feedstocks to biofuels. A mathematical model that considers both spatial and temporal phenomena in a biomass particle has the potential to accurately predict carbohydrate conversion and macroscopic structural changes during pretreatment. The development, implementation, and validation of a multiphysics model for high-solids (44\%) dilute-acid pretreatment of a preimpregnated corn-stover particle is presented. The model consists of tightly coupled time-dependent reaction-diffusion equations with finite-rate chemistry. The model partial-differential equations were discretized and solved numerically by the Legendre spectral finite element method. The simulation results were compared to experimental data that were obtained by performing pretreatment on pre-impregnated corn stover in a steam-explosion reactor. The majority of the modeling parameters were set by established science, obtained from the literature, or were experimentally measured initial conditions. The remaining unknown kinetic rate parameters were obtained by fitting simulation results to a limited set of experimental data. Reasonable
\end{abstract}

\footnotetext{
${ }^{*}$ Corresponding author

Email address: Jonathan.Stickel@nrel.gov (Jonathan J. Stickel)
} 
quantitative agreement was subsequently obtained between simulation results and a larger set of experimental data. The model simulations illustrate the strong dependence of the biomass conversion rate on the spatially dependent and transient acid concentration within the particle. It is observed that time scales of thermal-diffusion are much shorter than those for chemical reactions, which results in an isothermal condition for long pretreatment times. Diffusion of steam within the biomass particle dictates acid dilution and the zone where reactions occur.

Keywords: biofuels, lignocellulosic biomass, spectral finite element

method, reaction-diffusion equations

\section{Introduction}

The biochemical conversion of lignocellulosic biomass to alcohol or hydrocarbon fuels involves three distinct operations, namely, pretreatment, enzymatic hydrolysis, and fermentation. Pretreatment of biomass is performed mainly to reduce the resistance of biomass cell walls to enzymatic hydrolysis (so-called biomass recalcitrance) [1, 2]. Exposed cellulose chains in lignocellulosic materials after pretreatment are more easily hydrolyzed to sugars via enzymatic hydrolysis and are subsequently converted to ethanol by fermentation. Pretreatment can be performed using several mechanical and physicochemical methods that include vibratory ball milling [3], steam explosion [4], ammonia fiber expansion [5], dilute-acid hydrolysis [6, 7, 8], alkali hydrolysis $[9,10]$ and using ionic liquids [11].

Among these techniques, steam explosion disrupts the outer lignin structure, while dilute-acid pretreatment catalyzes the conversion of hemicellulose components such as xylan to simpler sugars [12]. A combination of these two methods, commonly called steam-assisted dilute-acid pretreatment, is a leading pretreatment process currently being developed in several laboratories. In its basic form, dilute-acid pretreatment involves the impregnation of milled biomass particles with sulfuric acid and subsequent rapid exposure of the 
biomass to saturated steam [13].

Acid-impregnated biomass particles undergoing steam explosion is a complex process that includes heat and mass transfer, steam-water phase transitions, and chemical reactions. Thermal-diffusion time scales are typically much shorter than chemical reactions and species mass transfer in the pretreatment processes. Acid-catalyzed hydrolysis tends to be strongly dependent on water content in the biomass particles due to dilution of local acid concentrations. Steam diffusion, condensation, and liquid evaporation contribute to the spatio-temporal variation of acid concentration in the particle. There are several mathematical models in the literature that studied the different aspects of pretreatment, including chemical kinetics, phase transitions, and diffusive transport. These models have been been used for biomass pretreatment, pulping processes in the paper industry, and also in food processing. We describe these models in the order of complexity with which the different aspects of pretreatment are addressed.

Modeling of pretreatment chemical kinetics have been performed by solving coupled ordinary-differential equations (ODEs) for concentrations of various participating species that include xylan, xylooligomers, xylose, and furfural. Esteghlalian et al. [6] studied detailed kinetics of dilute-acid pretreatment using first-order Arrhenius reaction rates. The rate constants were fit to experimental data for a simple analytically solved kinetics model, and optimum pretreatment conditions were determined for maximizing sugar yield. Mittal et al. [14] simulated hydrothermal pretreatment using coupled ODEs, where xylan undergoes autohydrolysis to yield xylooligomers, xylose, and furfural. The spatial variations of species concentrations via diffusive mass transport were neglected in this model and an average mass-transfer coefficient was used as a source term to account for non-reactive mass transfer. Gustafson et al. [15] developed a model for the kraft pulping process, which involves both diffusive mass transfer and chemical reactions. The pulping reactions consume alkali and release carbohydrates, lignin, and acetyl groups 
that diffuse out of the wood chips. The reduction in alkali concentration augments diffusive mass transfer from the bulk into the particle. The model was used to predict the time evolution of the chip lignin and carbohydrate content during the pulping reactions and the effective alkali concentration as a function of wood lignin content. Sensitivity of chip thickness to various metrics pertinent to the kraft pulping process, such as total yield, screened kappa number, and effective alkali consumption, was also explored using this model.

Abasaeed et al. [16] simulated acid hydrolysis of hardwood cellulose using a one-dimensional model comprised of both transient and spatially dependent heat transfer and chemical reactions. Typically, the wood particle is assumed to be isothermal when time scales of thermal conduction are much shorter than those of chemical reactions. The time scales become comparable for sufficiently large particles, and transient temperature effects become important. The model was used to investigate the effect of the chip size on sugar yield and reaction-completion times at different temperatures and acid concentrations.

Xu et al. [17] developed a steady-state model for heat and moisture transfer through textiles. This model included heat and mass transfer, phase transitions, and kinetics pertaining to condensation and evaporation. Coupled ODEs for temperature, water-vapor pressure, water-vapor mass flux through textile pores, and steam condensation on the surface of the textile were solved in this model. The mass transfer of water was modeled using approximate kinetic coefficients and not through diffusive transport.

Sprague and Colvin [18] developed a model that included heat and mass transfer, phase transitions, and chemical reactions for food-processing applications. Their model accounted for heat and liquid-water transport, evaporation of liquid water into steam, steam transport, and heterocyclic amine formation in a frying beef patty. It was used to simulate the spatio-temporal variation of temperature and to estimate the average heterocyclic amine con- 
centration in the patty as a function of the initial frying-pan temperature. A beef patty is a porous and fibrous medium like biomass and the modeling concepts employed are relevant to the multi-physics model developed in this paper.

The focus of this work was to develop a multiphysics model for an individual biomass (corn stover) particle that accounts for the above-mentioned transient reaction-diffusion processes for steam, various chemical species, and temperature. We combined several of the modeling concepts discussed above and develop additional components as needed based on our current understanding of the high-solids pretreatment process. Simulation results were validated with experimental results obtained from a steam-explosion reactor in which pre-impregnated biomass particles at high solids are fully fluidized and well mixed.

\section{Mathematical model}

\subsection{Assumptions}

The model assumes high-solids pretreatment of a pre-impregnated cylindrical biomass particle, with length $2 \ell$ and diameter $d$ as shown in Fig. 1, that consists of solid, liquid, and gas phases at any point during pretreatment. The representative mass and volume fractions, which can vary along the length of the particle are also shown. The gas phase, which is mainly steam, has negligible mass fraction but can occupy a major portion of the volume. The volume fractions of the three different phases in a biomass particle are related as

$$
\begin{gathered}
\epsilon_{P}+\epsilon_{S}=1, \\
\epsilon_{P}=\epsilon_{L}+\epsilon_{G},
\end{gathered}
$$

where $\epsilon_{P}, \epsilon_{S}, \epsilon_{L}$ and $\epsilon_{G}$ represent porosity, solid volume fraction, liquid volume fraction, and gas volume fraction in the particle, respectively. The heat and mass transfer are assumed to occur along the length of the particle. 


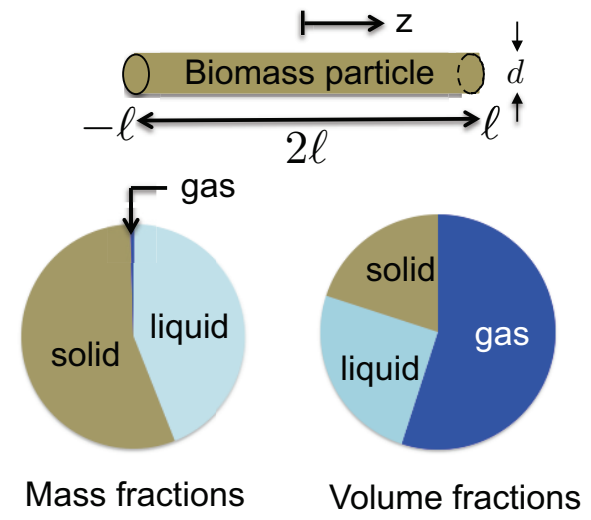

Figure 1: Cylindrical biomass particle with pie charts depicting typical mass and volume fractions of solid, liquid, and gas phases.

Plant biomass is known to have a porous structure that is highly anisotropic [19], and hence we assume a one-dimensional model for mass diffusion. On the other hand, transverse heat transfer will be higher due to large surface-area to volume ratios. The diameter $d$ is obtained from the length of the particle by assuming an aspect ratio $(2 \ell / d)$ of 20 . Quantitative optical measurements of milled corn stover particles indicated a range of aspect ratios between 1 and 30 [20]. A sensitivity of the current model to aspect ratio is also provided in section 6 .

The pretreatment process ideally involves the conversion of long-chain xylan into xylooligomers and ultimately into monomeric xylose. Xylose is further converted to the undesired degradation product furfural, which is the final product species assumed in this model. The chemical reaction schematic is shown in Fig. 2. All of the reactions are acid catalyzed, and we assume a homogeneous concentration of acid throughout the particle as a result of preimpregnation. The total amount of acid remains constant during pretreatment, while its molar concentration is reduced due to steam condensation and subsequent dilution. The mass of non-reacting solids, primarily lignin and glucan, is assumed to remain constant during pretreatment. 


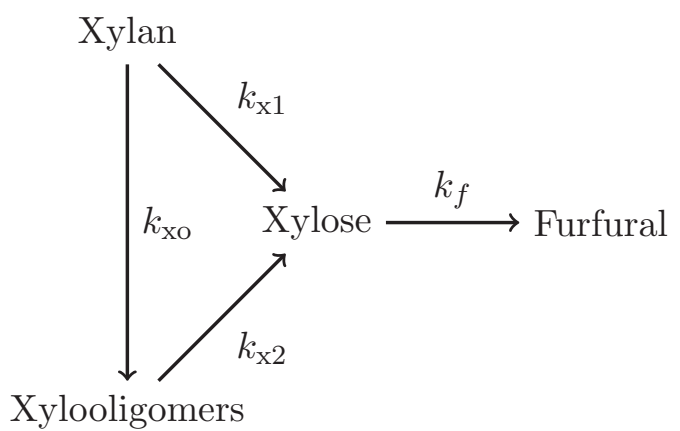

Figure 2: Chemical-reaction pathways for the pretreatment process along with symbols depicting reaction-rate constants.

\subsection{Governing equations}

The transport and volumetric loss of steam along the biomass particle is given by

$$
\frac{\partial c_{s}}{\partial t}=\frac{\partial}{\partial z}\left(D_{s} \frac{\partial c_{s}}{\partial z}\right)-k_{\text {cond }} c_{s}\left(\epsilon_{P}-\epsilon_{L}\right)+k_{\text {evap }}\left(\epsilon_{L}-\epsilon_{L T}\right) \frac{\rho_{L}}{M_{w}},
$$

where the first term on the right-hand side (RHS) represents diffusion, the second term represents the loss of steam through condensation, and the third term represents addition of steam via evaporation. The modeling approach for condensation and evaporation are discussed further in section 2.4. Here, $c_{s}$ represents the steam concentration and $D_{s}$ is the steam-diffusion coefficient; $\rho_{L}$ and $M_{w}$ represent water density and molecular weight, respectively; $k_{\text {cond }}$ and $k_{\text {evap }}$ are the condensation- and evaporation-rate constants, respectively; $\epsilon_{L T}$ represents the threshold liquid fraction above which liquid evaporation occurs. Further details regarding condensation and evaporation rate constants are discussed in section 2.4. The transient evolution of liquid volume fraction $\left(\epsilon_{L}\right)$ is given by

$$
\frac{\partial \epsilon_{L}}{\partial t}=\frac{M_{w} k_{\mathrm{cond}} c_{s}\left(\epsilon_{P}-\epsilon_{L}\right)}{\rho_{L}}-k_{\mathrm{evap}}\left(\epsilon_{L}-\epsilon_{L T}\right) .
$$

Liquid volume fraction increases via steam condensation and reduces via evaporation, represented by the two terms on the RHS. We neglected transport of liquid in the particle because the evaporation, steam transport, and 
condensation rates are expected to be faster than surface diffusion or capillary action.

The governing equation for the variation of xylan mass fraction in the solids $\tilde{f}_{x}$ is given by

$$
\frac{\partial \tilde{f}_{x}}{\partial t}=-\left(k_{\mathrm{xo}}+k_{\mathrm{x} 1}\right) \tilde{f}_{x} c_{\mathrm{acid}} \epsilon_{P}
$$

The reaction terms on the RHS represent the conversion of xylan to xylose and xylooligomers. The terms $k_{\mathrm{xo}}$ and $k_{\mathrm{x} 1}$ represent rate constants associated with conversion of xylan to xylooligomers and to xylose, respectively. The conversion rate also depends on the concentration of acid $c_{\text {acid }}$ and the porosity $\epsilon_{P}$ of the biomass particle. The governing equation for the variation of Xylooligomer concentration $\tilde{c}_{\text {xo }}$ is given by

$$
\frac{\partial \tilde{c}_{\mathrm{xo}}}{\partial t}=\frac{\partial}{\partial z}\left(D_{\mathrm{xo}} \frac{\partial c_{\mathrm{xo}}}{\partial z}\right)+k_{\mathrm{xo}} c_{\mathrm{acid}} \epsilon_{P}\left(\frac{M_{x}}{M_{\mathrm{xo}}}\right)\left(\frac{\tilde{f}_{x} \rho_{S}}{M_{x}}\right)-k_{\mathrm{x} 2} \tilde{c}_{\mathrm{xo}} c_{\mathrm{acid}} .
$$

The RHS of Eq. (5) includes diffusion of xylooligomers in solution and reaction terms associated with conversion to xylose and generation from xylan. Here, $D_{\text {xo }}$ represents the diffusion coefficient in solution of xylooligomers, $\rho_{S}$ represents the density of solid phase, and $k_{\mathrm{x} 2}$ is the rate constant of conversion of xylooligomers to xylose; $M_{x}$ and $M_{\text {xo }}$ are the molecular weights of xylan and xylooligomers, respectively. The governing equation for the variation of xylose concentration $\tilde{c}_{\mathrm{xy}}$ is given by

$$
\begin{array}{r}
\frac{\partial \tilde{c}_{\mathrm{xy}}}{\partial t}=\frac{\partial}{\partial z}\left(D_{\mathrm{xy}} \frac{\partial c_{\mathrm{xy}}}{\partial z}\right)+k_{\mathrm{x} 1} c_{\mathrm{acid}} \epsilon_{P}\left(\frac{M_{x}}{M_{\mathrm{xy}}}\right)\left(\frac{\tilde{f}_{x} \rho_{S}}{M_{x}}\right)-k_{f} \tilde{c}_{\mathrm{xy}} c_{\mathrm{acid}} \\
+k_{\mathrm{x} 2} \tilde{c}_{\mathrm{xo}} c_{\mathrm{acid}}\left(\frac{M_{\mathrm{xo}}}{M_{\mathrm{xy}}}\right) .
\end{array}
$$

Xylose is produced from the breakdown of xylan and xylooligomers and gets converted to furfural. It also diffuses in solution (with diffusion coefficient $D_{\text {xy }}$ ) through concentration gradients, represented by the first term on the RHS. The rate constant $k_{f}$ is associated with conversion of xylose to furfural and $M_{\mathrm{xy}}$ is the molecular weight of xylose. The governing equation for 
transient and spatial variation of furfural concentration $\tilde{c}_{f}$ is given by

$$
\frac{\partial \tilde{c}_{f}}{\partial t}=\frac{\partial}{\partial z}\left(D_{f} \frac{\partial c_{f}}{\partial z}\right)+k_{f} \tilde{c}_{\mathrm{xy}} c_{\mathrm{acid}}\left(\frac{M_{\mathrm{xy}}}{M_{f}}\right) .
$$

Furfural is the final product in the finite-rate chemistry shown in Fig. 2 and is formed from xylose. It is also a water-soluble compound and diffuses spatially within the biomass particle. The term $D_{f}$ represents the furfural diffusion coefficient in solution and $M_{f}$ is its molecular weight.

The thermal-energy equation associated with the common temperature $T$ associated with solid, liquid, and gas phases in the biomass particle is given by

$$
\begin{aligned}
\rho_{\mathrm{eff}} C_{\mathrm{eff}} \frac{\partial T}{\partial t}=\frac{\partial}{\partial z}\left(k_{\mathrm{eff}}\right. & \left.\frac{\partial T}{\partial z}\right)+\frac{4 h}{d}\left(T_{s}-T\right) \\
& +L\left[k_{\mathrm{cond}} c_{s}\left(\epsilon_{P}-\epsilon_{L}\right) M_{w}-k_{\text {evap }}\left(\epsilon_{L}-\epsilon_{L T}\right) \rho_{L}\right],
\end{aligned}
$$

where $C_{\text {eff }}, \rho_{\text {eff }}$ and $k_{\text {eff }}$ represent the volume-weighted effective specific heat, density, and thermal conductivity, respectively. The temperature varies spatially within the particle via thermal-diffusion and the latent heat $(L)$ source term on the RHS of Eq. (8). The temperature variation along the radial direction is neglected in the model, but the heat transfer through the lateral surface of the particle is accounted for by a source term on the RHS. This depends on the convective heat transfer coefficient $h$ and varies linearly with the temperature difference between the particle interior and the ambient surrounding steam temperature $\left(T_{s}\right)$. The lateral heat transfer is directly proportional to the surface-area to volume ratio, which depends on the reciprocal of particle diameter $d$.

\subsection{Transport coefficients}

The diffusion coefficient of steam can be obtained from the Knudsen diffusion formula $[21,22]$ given by

$$
\begin{gathered}
D_{s}^{0}=\frac{d_{\text {pore }}}{3} \sqrt{\frac{8 k_{B} T}{\pi M_{w}}}, \\
D_{s}=\left(\epsilon_{P}-\epsilon_{L}\right) D_{s}^{0},
\end{gathered}
$$


where $k_{B}$ is the Boltzmann's constant and $T$ is the temperature of the biomass particle. The pore diameter $d_{\text {pore }}$ in pretreated corn stover found in literature ranges from 2 to $20 \mathrm{~nm}$ [23]. Mean free path of steam at pretreatment temperatures and pressures of approximately $400 \mathrm{~K}$ and 100 psi, respectively, is approximately $35 \mathrm{~nm}$ for water molecule size of $0.3 \mathrm{~nm}$. The pore diameters are therefore comparable to steam mean free paths, making the Knudsen diffusion assumption valid for the current study. The steam-diffusion coefficient is also scaled by $\epsilon_{G}=\epsilon_{P}-\epsilon_{L}$, which is the fraction of accessible pores for the gas phase.

Diffusion coefficients of solutes (xylose, furfural, and xylooligomers) are obtained through the Stokes-Einstein diffusion law,

$$
\begin{aligned}
D^{0} & =\frac{k_{B} T}{6 \pi \eta r}, \\
D & =\epsilon_{L} D^{0} .
\end{aligned}
$$

Here, $\eta$ is the dynamic viscosity of the solvent (water), and $r$ is the molecular radius of the solute molecule. The solute diffusion coefficient is also scaled by the liquid volume fraction $\epsilon_{L}$. The molecular diameter of xylose and furfural are reported to be $0.68 \mathrm{~nm}$ and $0.57 \mathrm{~nm}$, respectively [24]. The size of xylooligomers depends on the number of xylose molecules linked together in the oligomer. This includes Xylobiose (2 molecules), Xylotriose (3), Xyloetrose (4), and Xylopentose (5) [25]. Xylotriose is assumed to be the dominant oligomer in the chemistry used in the simulations. For water at $100^{\circ} \mathrm{C}, \eta=2.8 \times 10^{-4} \mathrm{~Pa} \mathrm{~s}$.

\subsection{Reaction rates}

The rate coefficients used in the finite-rate chemistry are in the first-order Arrhenius form given by

$$
k=A \exp \left(-E_{a} / R T\right)
$$

where $A$ is the pre-exponential factor and $E_{a}$ is the activation energy. 
The rate coefficients for steam evaporation and condensation depend on the local temperature of the biomass particle. While the steam outside the particle is constant at its saturation temperature, local temperatures within the particle fluctuate due to heat transfer and phase change. The saturation temperature of steam $\left(T_{s}\right)$ is assumed to be the threshold above which the steam condensation rate becomes zero and below which the steam evaporation rate becomes zero. When condensation or evaporation conditions are met, it is assumed to occur at a constant rate of $\bar{k}=10 \mathrm{~s}^{-1}$, which was used by Sprague and Colvin [18] and Nguyen and White [26]. The evaporation rate constant also has a threshold liquid fraction below which evaporation does not take place. This threshold $\epsilon_{L T}$ is assumed to be the total initial porosity of the biomass particle. It is assumed that liquid evaporation happens only after all the pores (locally, at position $x$ ) are filled. Sprague and Colvin [18] assumed the threshold to be the initial liquid fraction, which was the same as the total porosity. The condensation- and evaporation-rate constants are given by

$$
\begin{gathered}
k_{\text {cond }}= \begin{cases}\bar{k}, & \text { if } T \leq T_{s}, \\
0, & \text { if } T>T_{s},\end{cases} \\
k_{\text {evap }}= \begin{cases}\bar{k}, & \text { if }\left(T \geq T_{s}\right) \text { and }\left(\epsilon_{L}>\epsilon_{L T}\right), \\
0, & \text { otherwise. }\end{cases}
\end{gathered}
$$

The variation with temperature of both rate constants are implemented in the simulation code with linear variation within $0.1 \%$ of the saturation temperature instead of a sharp discontinuity.

\subsection{Boundary conditions}

Only half of the biomass particle illustrated in Fig. 1 is simulated, i.e., $x \in[0, \ell]$, assuming symmetry at $x=0$. The boundary conditions for Eqs. (2), (5)-(8) are shown in Table 1. The gradients of all variables vanish at $x=0$ to ensure symmetry. The species gradients for xylooligomers, xylose and furfural go to zero at the boundaries thereby enforcing a zero-flux 
Table 1: Boundary conditions for the governing equations.

\begin{tabular}{cc}
\hline \hline$x=0$ & $x=\ell$ \\
\hline$\frac{\partial c_{s}}{\partial z}=0$ & $c_{s}=c_{s, \text { bulk }}$ \\
$\frac{\partial c_{\mathrm{xo}}}{\partial z}=0$ & $\frac{\partial c_{\mathrm{xo}}}{\partial z}=0$ \\
$\frac{\partial c_{\mathrm{xy}}}{\partial z}=0$ & $\frac{\partial c_{\mathrm{xy}}}{\partial z}=0$ \\
$\frac{\partial c_{f}}{\partial z}=0$ & $\frac{\partial c_{f}}{\partial z}=0$ \\
$\frac{\partial T}{\partial z}=0$ & $-k_{\mathrm{eff}} \frac{\partial T}{\partial z}=h\left(T-T_{s}\right)$ \\
\hline \hline
\end{tabular}

condition. The ambient steam concentration $\left(c_{s, \mathrm{bulk}}\right)$ in the reactor is applied as a Dirichlet boundary condition at $x=\ell$. A Robin-type boundary condition is used for the thermal-energy equation, where thermal conduction flux within the particle is matched by convective heat transfer of ambient steam. Eqs. (3) and (4) do not have diffusion terms and do not require boundary conditions. Their initial values are obtained from experimental data.

\subsection{Other relations}

Xylan and water-soluble species (xylooligomers, xylose, and furfural) concentrations are relative to the volume of solid and water in the biomass particle, which change with steam condensation and porosity variation. Therefore, the species governing equations (Eqs. (4)-(7)) have to be formulated on an absolute molar basis to ensure mass balance in simulations (i.e., the tilde denotes dimensions of [mole per particle volume] rather than [mole per liquid volume] or [mole per solid volume]). The dependent variables in the 
governing equations are given by

$$
\begin{array}{r}
\tilde{f}_{x}=f_{x}\left(1-\epsilon_{P}\right), \\
\tilde{c}_{\mathrm{xo}}=c_{\mathrm{xo}} \epsilon_{L}, \\
\tilde{c}_{\mathrm{xy}}=c_{\mathrm{xy}} \epsilon_{L}, \\
\tilde{c}_{f}=c_{f} \epsilon_{L},
\end{array}
$$

where the dependent variables in the governing equations are obtained by scaling the molar concentrations with the corresponding volume fractions. Xylan mass fraction is scaled by the solid volume fraction, while the liquid soluble compounds are scaled by the liquid volume fraction. The initial condition for the equation governing xylan mass fraction (Eq. (4)) is given by

$$
\tilde{f}_{x}^{0}=f_{x}^{0}\left(1-\epsilon_{P}^{0}\right)
$$

where a zero superscript denotes an initial condition, e.g. $f_{x}^{0}$ is the initial xylan mass fraction. The porosity of the particle at any spatial location is determined by assuming that solid components other than xylan do not undergo conversion. The governing equation for porosity can be formulated as follows. Consider a volume $\delta V$ of the biomass particle with a solid mass $m_{T}$ and porosity $\epsilon_{P}$. The mass of xylan $m_{X}$ and other solids $m_{O S}$ is given by

$$
\begin{array}{r}
m_{X}=m_{T} f_{X}, \\
m_{O S}=m_{T}\left(1-f_{X}\right) .
\end{array}
$$

The total solid volume $\left(\left(1-\epsilon_{P}\right) \delta V\right)$ can be written in terms of the mass of other solids and densities of xylan $\left(\rho_{X}\right)$ and other solids $\left(\rho_{O S}\right)$ as

$$
\frac{m_{O S} f_{X}}{\rho_{X}\left(1-f_{X}\right)}+\frac{m_{O S}}{\rho_{O S}}=\left(1-\epsilon_{P}\right) \delta V .
$$

Because $m_{O S}$ and $\delta V$ are invariant over time, taking the ratio Eq. (20) for time $t=0$ and at time $t$, we get the governing equation for porosity. 


$$
\frac{\frac{f_{X}^{0}}{1-f_{X}^{0}}+\frac{\rho_{X}}{\rho_{O S}}}{1-\epsilon_{P}^{0}}=\frac{\frac{f_{X}}{1-f_{X}}+\frac{\rho_{X}}{\rho_{O S}}}{1-\epsilon_{P}}
$$

The effective specific heat, mass density, and thermal conductivity terms used in Eq. (8) are obtained as volume-fraction-weighted average of values corresponding to solid, liquid, and gas phases as

$$
\begin{array}{r}
\rho_{\mathrm{eff}} C_{\mathrm{eff}}=\left(1-\epsilon_{P}\right) \rho_{S} C_{S}+\epsilon_{L} \rho_{L} C_{L}+\epsilon_{G} \rho_{G} C_{G}, \\
k_{\mathrm{eff}}=\left(1-\epsilon_{P}\right) k_{S}+\epsilon_{L} k_{L}+\epsilon_{G} k_{G} .
\end{array}
$$

Here $C, \rho$ and $k$ with solid $(S)$, liquid $(L)$ and gas $(G)$ subscripts represent individual phase specific heat, mass density and thermal conductivity, respectively. Because the acid is assumed to be a catalyst, the total number of moles does not change. Therefore, the acid concentration decreases with increase in liquid fraction as

$$
c_{\text {acid }} \epsilon_{L}=c_{\text {acid }}^{0} \epsilon_{L}^{0}
$$

\section{Numerical method}

Eqs. (2)-(8) represent the transient multiphase reaction-diffusion equations for the one-dimensional pretreatment model. These equations are discretized in space using the Legendre spectral finite element (LSFE) method [27]. Spectral finite element methods can provide optimal solutions with a minimum number of degrees of freedom and are well suited for highly regular solutions that arise in reaction-diffusion systems. When combined with nodal quadrature, LSFEs also have diagonal mass matrices, which are easily inverted for time-stepping methods. The system of partial-differential equations can be generalized into a strong form as

$$
\begin{array}{r}
\frac{\partial \mathbf{u}}{\partial t}+\frac{\partial}{\partial z}([\mathbf{C}] \mathbf{u})=\frac{\partial}{\partial z}\left([\mathbf{D}] \frac{\partial \mathbf{u}}{\partial z}\right)+[\mathbf{R}] \mathbf{u}+\mathbf{f} \\
\mathbf{u}=\left[\begin{array}{lllllll}
c_{s} & \epsilon_{L} & \tilde{f}_{x} & \tilde{c}_{\mathrm{xo}} & \tilde{c}_{\mathrm{xy}} & \tilde{c}_{f} & T
\end{array}\right]^{T},
\end{array}
$$


where $\mathbf{u}$ is the solution vector consisting of seven variables, and the $T$ superscript denotes a transpose.

The terms $[\mathbf{C}],[\mathbf{D}]$ and $[\mathbf{R}]$ are diagonal matrices that represent convection, diffusion and reaction terms respectively, and are given by

$$
\begin{gathered}
{[\mathbf{C}]=\operatorname{diag}\left[\begin{array}{c}
0 \\
0 \\
0 \\
\frac{D_{\mathrm{xo}}}{\epsilon_{L}} \frac{\partial \epsilon_{L}}{\partial z} \\
\frac{D_{\mathrm{xy}}}{\epsilon_{L}^{2}} \frac{\partial \epsilon_{L}}{\partial z} \\
\frac{D_{\mathrm{f}}}{\epsilon_{L}^{2}} \frac{\partial \epsilon_{L}}{\partial z} \\
0
\end{array}\right],[\mathbf{D}]=\operatorname{diag}\left[\begin{array}{c}
D_{s} \\
0 \\
0 \\
\frac{D_{\mathrm{xo}}}{\epsilon_{L}} \\
\frac{D_{\mathrm{xy}}}{\epsilon_{L}} \\
\frac{D_{\mathrm{f}}}{\epsilon_{L}} \\
\frac{k_{\mathrm{eff}}}{\rho_{\mathrm{eff}} C_{\mathrm{eff}}}
\end{array}\right],} \\
{[\mathbf{R}]=\operatorname{diag}\left[\begin{array}{c}
-k_{\mathrm{cond}}\left(\epsilon_{P}-\epsilon_{L}\right) \\
-\frac{M_{w}}{\rho_{L}} k_{\mathrm{cond}} c_{s}-k_{\mathrm{evap}} \\
-\left(k_{\mathrm{xo}}+k_{\mathrm{x} 1}\right) c_{\mathrm{acid}} \epsilon_{P} \\
-k_{\mathrm{x} 2} c_{\mathrm{acid}} \\
-k_{\mathrm{f}} c_{\mathrm{acid}} \\
0 \\
\frac{-4 h}{\rho_{\mathrm{eff}} C_{\mathrm{eff}}}
\end{array}\right]}
\end{gathered}
$$

The convection terms for soluble species in Eq. (27) arise due to the formulation of thermodynamically consistent diffusive flux for which the gradient of concentration with respect to water $\left(c_{\mathrm{xo}}, c_{\mathrm{xy}}, c_{\mathrm{f}}\right)$ is used rather than the absolute molar concentration $\left(\tilde{c}_{\mathrm{xo}}, \tilde{c}_{\mathrm{xy}}, \tilde{c}_{\mathrm{f}}\right)$. 
The source term $\mathbf{f}$ is given by

$$
\mathbf{f}=\left[\begin{array}{c}
k_{\mathrm{evap}}\left(\epsilon_{L}-\epsilon_{L T}\right) \frac{\rho_{L}}{M_{w}} \\
k_{\mathrm{cond}} c_{s} \epsilon_{P} \frac{M_{w}}{\rho_{L}}+k_{\mathrm{evap}} \epsilon_{L T} \\
0 \\
k_{\mathrm{xo}} \rho_{S} c_{\mathrm{acid}} \epsilon_{P} \frac{\tilde{f}_{x}}{M_{\mathrm{xo}}} \\
k_{\mathrm{x} 1} \rho_{S} c_{\mathrm{acid}} \epsilon_{P} \frac{\tilde{f}_{x}}{M_{\mathrm{xy}}}+k_{\mathrm{x} 2} \tilde{c}_{\mathrm{xo}} c_{\mathrm{acid}} \frac{M_{\mathrm{xo}}}{M_{\mathrm{xy}}} \\
k_{f} \tilde{c}_{\mathrm{xy}} c_{\mathrm{acid}} \frac{M_{\mathrm{xy}}}{M_{f}} \\
\frac{L}{\rho_{\mathrm{eff}} C_{\mathrm{eff}}}\left(k_{\mathrm{cond}} c_{s}\left(\epsilon_{P}-\epsilon_{L}\right) M_{w}-k_{\mathrm{evap}}\left(\epsilon_{L}-\epsilon_{L T}\right) \rho_{L}\right)+\frac{4 h}{\rho_{\mathrm{eff}} C_{\mathrm{eff}}} T_{s}
\end{array}\right]
$$

Each variable in the solution vector can be written as a linear combination of Lagrangian-interpolant finite-element basis functions.

The original set of equations (Eq. (26)), after spatial integration and subsequent weakening, result in coupled ODEs with nodal values $\mathbf{U}$ as the dependent variable, given by

$$
[\mathbf{M}] \frac{d \mathbf{U}}{d t}+\left[\mathbf{K}_{\mathbf{C}}\right] \mathbf{U}=\left[\mathbf{K}_{\mathbf{D}}\right] \mathbf{U}+\left[\mathbf{K}_{\mathbf{R}}\right] \mathbf{U}+\mathbf{F}
$$

where $[\mathbf{M}]$ represents the mass matrix, $\left[\mathbf{K}_{\mathbf{C}}\right]$ represents the convection stiffness matrix, $\left[\mathbf{K}_{\mathbf{D}}\right]$ represents the diffusion stiffness matrix, $\left[\mathbf{K}_{\mathbf{R}}\right]$ represents the reaction stiffness matrix, and $\mathbf{F}$ is the source term. The coupled ODEs are solved using a semi-implicit time stepping method to circumvent the severe time step restrictions imposed by steam and temperature diffusion time scales.

The diffusion coefficients for steam and temperature equations tend to be 1000 to 10,000 times larger than species diffusion making the system extremely stiff. The semi-implicit time integration scheme can be formulated as

$$
[\mathbf{M}]\left(\frac{\mathbf{U}^{n+1}-\mathbf{U}^{n}}{\delta t}\right)+\left[\mathbf{K}_{\mathbf{C}}\right]^{n} \mathbf{U}^{n+1}=\left[\mathbf{K}_{\mathbf{D}}\right]^{n} \mathbf{U}^{n+1}+\left[\mathbf{K}_{\mathbf{R}}\right]^{n} \mathbf{U}^{n+1}+\mathbf{F}^{n}
$$

where the superscript $n$ denotes the time-level and the total time is given by $t^{n}=n \Delta t$, where $\Delta t$ is the time interval. First-order backward-difference time stepping is used with the right-hand side of Eq. (29) written at the future 
time level $n+1$, with approximate linearization by time-lagging the stiffness matrices. This approach requires a linear-system solve at every time step, which is done through a matrix-free generalized minimum residual (GMRes) algorithm. The simulation results shown in section 5 are obtained using a mesh of 15 elements with a polynomial order of three. The time increment used for the implicit time stepping is $0.25 \mathrm{~s}$, which was sufficiently accurate when compared to a fourth-order Runge-Kutta simulation with a time-step of $0.0002 \mathrm{~s}$ that corresponds to steam diffusion time scales. The solutions were also tested with more degrees of freedom and were found to be grid independent. Simulations were run on a single modern cpu and simulations to one minute required about three minutes of wall-clock time.

\section{Bench-scale pretreatment experiments}

\subsection{Feedstock and dilute-acid impregnation}

Pioneer maize variety 33A14 whole corn stover grown in Wray, Colorado was used for the experimental work. Corn-stover particle size was reduced to pass a $6.4 \mathrm{~mm}$ (0.25 in.) screen using a knife mill. Feedstock processing and storage has been previously described by Shekiro et al. [28]. Milled corn stover was subsequently pre-impregnated with acid by soaking in a recirculating acid bath [13]. For this study, $10 \mathrm{~kg}$ of corn stover (dry-basis) was soaked at $7 \%(\mathrm{w} / \mathrm{w})$ solids for two hours in a Hastelloy mesh basket. After two hours the basket was lifted from the acid solution and allowed to drain until a small amount of liquid was dripping from the biomass. After draining of the acid, the soaked slurry was $20 \%$ (w/w) solids. The post-drain slurry was further dewatered to $44 \%$ (w/w) solids using a hydraulic piston press [28]. At this solids concentration, all moisture was contained within the pores of the biomass particles. The acid impregnated biomass (AIB) was

stored at $4^{\circ} \mathrm{C}$ until it was pretreated. Solids composition of the AIB was measured, and is shown in table 2 . 
Table 2: Solids composition of acid-impregnated biomass at the acid $\left(\mathrm{H}_{2} \mathrm{SO}_{4}\right)$ concentrations indicated.

\begin{tabular}{ccc}
\hline \hline Compound & $0.5 \%(\mathrm{w} / \mathrm{w})$ & $1.0 \%(\mathrm{w} / \mathrm{w})$ \\
\hline Glucan & 42.3 & 41.6 \\
Xylan & 24.8 & 26.3 \\
Galactan & 1.4 & 1.3 \\
Arabinan & 3.7 & 3.7 \\
Acetate & 2.9 & 3.0 \\
Lignin & 18.1 & 18.1 \\
Ash & 3.5 & 3.4 \\
\hline \hline
\end{tabular}

\subsection{Pretreatment and analysis}

Dilute-acid pretreatment was performed in the National Renewable energy Laboratory (NREL) batch steam explosion reactor, which is described elsewhere [13]. Briefly, the batch reactor has a total volume of $4 \mathrm{~L}$, and is heated by direct injection of saturated steam. The reactor was loaded with $500 \mathrm{~g}$ of AIB at a solids fraction of 0.44 for each pretreatment run. Over the course of pretreatment, steam condenses, causing a transient dilution profile of components solubilized during pretreatment. When the desired time was reached $(5,10,20,30$, and $40 \mathrm{~min}$ in this study), the reaction was quenched by quickly discharging into a 55 gallon polypropylene bag in a process known as steam explosion. Vapors were allowed to condense in the bag and the resulting slurry is stored at $4^{\circ} \mathrm{C}$ until analysis.

For this study, AIB at $0.5 \%(\mathrm{w} / \mathrm{w}) \mathrm{H}_{2} \mathrm{SO}_{4}$ and $1.0 \% \mathrm{H}_{2} \mathrm{SO}_{4}$ was pretreated at $150^{\circ} \mathrm{C}$ and $170^{\circ} \mathrm{C}$ at varied residence times. Two $500 \mathrm{~g}$ pretreatment runs were performed and collected in the same bag at each condition. A list of the pretreatment conditions is shown in table 3. Fraction insoluble solids (FIS), solids compositions, and monomeric and total sugar concentrations were determined using NREL's standard laboratory analytical procedures [29, 30]. 
Table 3: Biomass pretreatment experiments studied in this work. All experiments started with the same solids concentration (44\% FIS).

\begin{tabular}{cc}
\hline \hline$\left[\mathrm{H}_{2} \mathrm{SO}_{4}\right] \%(\mathrm{w} / \mathrm{w})$ & Temperature $\left({ }^{\circ} \mathrm{C}\right)$ \\
\hline 0.5 & 150 \\
0.5 & 170 \\
1.0 & 150 \\
1.0 & 170 \\
\hline \hline
\end{tabular}

\section{Results}

\subsection{Base-case simulation parameters}

Table 4 gives the initial conditions that were used for what we specify to be the "base-case" simulations, i.e., those that were for the initial conditions of saturated steam at $150^{\circ} \mathrm{C}$ and the biomass impregnated with $1 \%$ acid by weight. The initial conditions for the other simulations were set to match the corresponding experimental conditions and varied accordingly from those shown in Table 4 . Table 5 gives the model parameters that were invariant between the set of simulations performed in this work (excluding the kinetic rate parameters that are discussed below). The solid density $\left(\rho_{s}\right)$ is assumed to be a nominal value of $1000.0 \mathrm{~kg} / \mathrm{m}^{3}$, obtained from true density measurements of biomass feedstocks reported in literature [31].

The density of solids other than xylan $\left(\rho_{O S}\right)$ is assumed to be an average density of dominant solids, glucan $\left(\rho_{G l}\right)$ and lignin $\left(\rho_{L i}\right)$. The liquid density $\left(\rho_{l}\right)$ is assumed to be that of water and its marginal variation with temperature is neglected. The particle length $(2 \ell)$ is assumed to be equal to $5 \mathrm{~mm}$ for all the simulation cases, for which a sensitivity study is performed in section 6 . The simulation results are compared with experimental measurements of species concentrations, water dilution via steam, and fraction of insoluble solids (FIS or $f_{i s}$ ) in the resulting slurry after various pretreatment times. The spatially varying species concentrations, water content, and xy- 
lan mass fractions from the simulations are averaged over the length of the particle to obtain time-dependent integrated parameters for the comparison study. The time-dependent spatially averaged soluble species concentrations for xylooligomers, xylose and furfural are given by

$$
\bar{c}_{i}=\frac{\int_{0}^{\ell} \tilde{c}_{i} \mathrm{~d} x}{\int_{0}^{\ell} \epsilon_{L} \mathrm{~d} x} .
$$

The dilution via steam $\bar{d}$ is measured as the amount of water added per gram of initial liquid, which is obtained from the simulations as

$$
\bar{d}=\frac{\int_{0}^{\ell} \epsilon_{L} \mathrm{~d} x-\epsilon_{L}^{0} \ell}{\epsilon_{L}^{0} \ell} .
$$

The integrated xylan mass fraction $\bar{X}$ in the solids is obtained as

$$
\bar{X}=\frac{\int_{0}^{\ell} \tilde{f}_{x} \mathrm{~d} x}{\int_{0}^{\ell}\left(1-\epsilon_{P}\right) \mathrm{d} x} .
$$

The expression for fraction of insoluble solids is obtained by first calculating the mass of solid phase $\left(\bar{m}_{S}\right)$ and liquid phase $\left(\bar{m}_{L}\right)$ in the biomass particle and finding the solid fraction, given by

$$
\begin{aligned}
\bar{m}_{S} & =\int_{0}^{\ell} \rho_{S}\left(1-\epsilon_{P}\right) \mathrm{d} x \\
\bar{m}_{L} & =\int_{0}^{\ell} \rho_{L} \epsilon_{L} \mathrm{~d} x \\
f_{i s} & =\frac{\bar{m}_{S}}{\bar{m}_{S}+\bar{m}_{L}} .
\end{aligned}
$$

The activation energies $\left(E_{a}\right)$ and pre-exponential factors $(A)$ used for the finite-rate chemistry are shown in Table 6. The activation-energy values were obtained from the literature $[6,40]$. The four pre-exponents were simultaneously fit to experimental data for the base-case simulations. Figure 3 compares experimental data for six different experimentally measured quantities with simulations. Although the good agreement for this particular data set is partly a result of parameter fitting of pre-exponential factors, it also indicates that the model captures the relevant chemical and physical 
Table 4: Initial conditions

\begin{tabular}{clc}
\hline \hline Parameter & Description & Value \\
\hline$c_{\text {acid }}^{0}$ & Initial acid concentration $(\mathrm{M})$ & 0.1 \\
$c_{s, \text { bulk }}$ & Ambient steam concentration $(\mathrm{M})$ & 0.14 \\
$f_{x}^{0}$ & Initial xylan mass fraction & 0.26 \\
$\epsilon_{P}^{0}$ & Initial porosity & 0.8 \\
$\epsilon_{L}^{0}$ & Initial liquid fraction & 0.25 \\
$f_{i s}^{0}$ & Initial fraction of insoluble solids & 0.44 \\
$T^{0}$ & Initial temperature field (K) & 300 \\
$T_{s}$ & Saturated steam temperature $(\mathrm{K})$ & 423 \\
\hline \hline
\end{tabular}

phenomena of pretreatment. Some discrepancies exist that may be partly the consequence of the simplifying assumptions used in the model, but it may also be the result of experimental uncertainty. We did not have sufficient replicate data to provide quantitative error bars for each data point. However, it is possible to calculate a mass balance around the solid species (insoluble and soluble) and xylan species (xylan, xylooligomers, xylan, and furfural), and the magnitude of the deviation of the mass balance from unity provides a quantitative indication of the uncertainty of the data. The massbalance deviation is shown as bars in the figure. The same set of model parameters are used to predict pretreatment results for three other sets of experiments, as discussed in the following section.

\subsection{Model validation}

Model simulation results are further compared to data from three additional sets of pretreatment experiments. Figure 4 shows the comparison of simulations with experiments for a case with $150^{\circ} \mathrm{C}$ saturated steam and $0.5 \%$ acid. The xylose concentration, water addition, and FIS have been predicted well, but the model under predicts the oligomer and furfural concentrations. The dilution in the experiment shows significant variability due to 
Table 5: Diffusion coefficients, thermal data and molecular weights used in the simulations.

\begin{tabular}{|c|c|c|c|}
\hline Parameter & Description & Value & Source \\
\hline \multicolumn{4}{|c|}{ Diffusion coefficient parameters (nm) } \\
\hline$d_{\text {pore }}$ & Pore diameter & 15 & {$[23]$} \\
\hline$r_{\mathrm{xy}}$ & xylose molecular radius & 0.34 & {$[24]$} \\
\hline$r_{\mathrm{xo}}$ & xylooligomer molecular radius & 1.02 & {$[24]$} \\
\hline$r_{f}$ & furfural molecular radius & 0.28 & {$[24]$} \\
\hline \multicolumn{4}{|c|}{ Thermal conductivities $(\mathrm{W} / \mathrm{m} / \mathrm{K})$} \\
\hline$k_{S}$ & Solid & 0.12 & {$[32]$} \\
\hline$k_{L}$ & Liquid & 0.63 & {$[33]$} \\
\hline$k_{G}$ & Gas & 0.0197 & {$[33]$} \\
\hline$h$ & Convective heat transfer coeff. (W/m²/K) & 5000.0 & {$[34]$} \\
\hline \multicolumn{4}{|c|}{ Specific heat capacities $(\mathrm{J} / \mathrm{kg} / \mathrm{K})$} \\
\hline$C_{S}$ & Solid & 2000.0 & {$[35]$} \\
\hline$C_{L}$ & Liquid & 4350.0 & {$[36]$} \\
\hline$C_{G}$ & Gas & 1970.0 & {$[36]$} \\
\hline \multicolumn{4}{|c|}{ Mass densities $\left(\mathrm{kg} / \mathrm{m}^{3}\right)$} \\
\hline$\rho_{l}$ & Liquid density & 1000.0 & $a$ \\
\hline$\rho_{s}$ & Solid density & 1000.0 & {$[31]$} \\
\hline$\rho_{X}$ & Xylan density & 730.0 & {$[37]$} \\
\hline$\rho_{G l}$ & Glucan density & 1500.0 & {$[38]$} \\
\hline$\rho_{L i}$ & Lignin density & 1300.0 & {$[39]$} \\
\hline \multicolumn{4}{|c|}{ Molecular weights $(\mathrm{g} / \mathrm{mol})$} \\
\hline$M_{x}$ & xylan molecular weight & 132 & $a$ \\
\hline$M_{\mathrm{xy}}$ & xylose molecular weight & 150 & $a$ \\
\hline$M_{\mathrm{xo}}$ & xylooligomer molecular weight & 450 & $a$ \\
\hline$M_{f}$ & furfural molecular weight & 96 & $a$ \\
\hline
\end{tabular}


Table 6: Arrhenius parameters for the finite-rate chemistry.

\begin{tabular}{clcc}
\hline \hline Rate Coeff. & Description & $A\left(\mathrm{~cm}^{3} / \mathrm{mol} / \mathrm{s}\right)^{a}$ & $E_{a}(\mathrm{~kJ} / \mathrm{mol})^{b}$ \\
\hline$k_{\mathrm{xo}}$ & xylan to xylooligomers & $1.0 \times 10^{15}$ & 110.0 \\
$k_{\mathrm{x} 1}$ & xylan to xylose & $8.0 \times 10^{17}$ & 130.0 \\
$k_{\mathrm{x} 2}$ & xylooligomers to xylose & $2.5 \times 10^{14}$ & 110.0 \\
$k_{f}$ & xylan to furfural & $7.0 \times 10^{11}$ & 98.0 \\
\hline \hline
\end{tabular}

${ }^{a}$ Obtained by fitting to experimental data.

${ }^{b}$ Obtained from literature $[6,40]$.
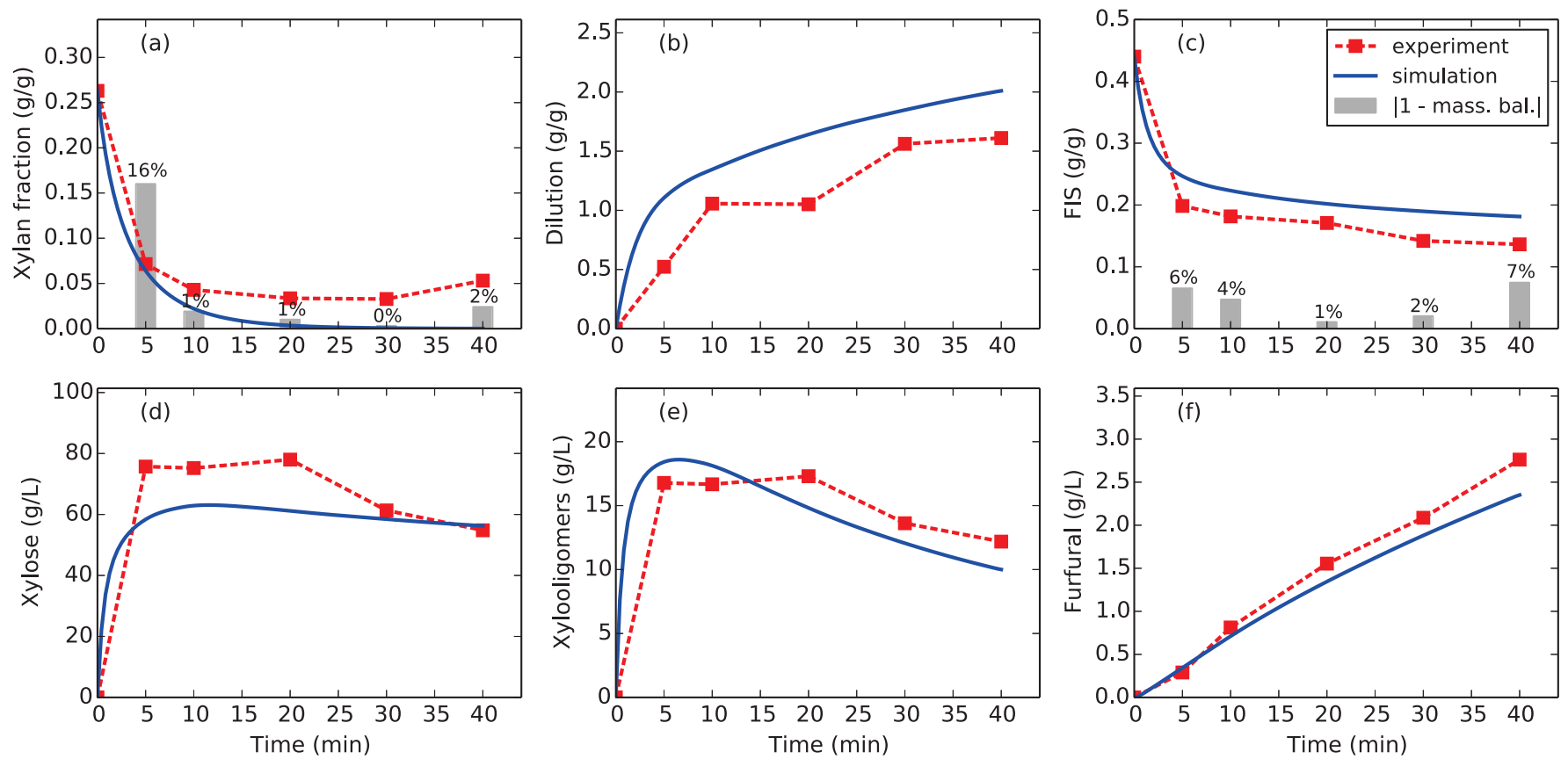

Figure 3: Experimentally measured and simulation-derived quantities after different times of pretreatment for the base-case conditions of $150^{\circ} \mathrm{C}$ steam and $1 \%$ acid: (a) mass fraction of xylan in the biomass solids, (b) amount of water added per gram of initial liquid, (c) mass fraction of insoluble solids in the slurry; soluble species concentrations (d) xylose, (e) xylooligomers, and (f) furfural. The bars show the magnitude of the deviation of the xylan-species mass balance (a) and solids mass balance (c) from unity, and hence the bars provide a quantitative indication of data uncertainty. 
measurement inaccuracies, which potentially affects the species concentration measurements. The xylan mass fraction diminish rapidly in the simulations compared to the experiments, reaching $99 \%$ conversion at the end of 40 minutes. Figure 5 shows the comparison for pretreatment using $170^{\circ} \mathrm{C}$ saturated steam and $1 \%$ acid. The pretreatment conditions in this case is where the biomass is exposed to highest temperature and highest concentration of acid, which results in the fastest conversion rates among the four experiments. The oligomer and furfural concentrations are slightly over predicted for this case while other quantities compare well. The simulation predicts peak oligomer concentrations at about 1 minute which is not resolved in the experimental sample times. Figure 6 compares the simulations and experiments for pretreatment using $170^{\circ} \mathrm{C}$ and $0.5 \%$ acid. All of the quantities tend to agree well with experiments. The xylose concentration is slightly over predicted by the model, while the xylooligomer and furfural concentrations are slightly under-predicted.

The model tends to capture a slightly higher FIS for all four cases studied. Depolymerization of only xylan is considered in the model while keeping the mass of other solids constant. This is not the case in the physical experiments where other components such as lignin and glucan are also know to be converted to soluble species during pretreatment. The dominant xylooligomer is assumed to be xylotriose in the simulations. This could be the reason for oligomer concentration deviations seen in Fig. 4. Significant deviation between predicted and measured furfural is observed in Fig. 4(f), which is the weakest pretreatment conditions studied among the four cases. This could be attributed to measurement errors in dilution (Fig. 4(b)), which may be comparatively lower than actual values, resulting in higher concentration levels. At strong pretreatment conditions, the furfural concentration is predicted to increase even after complete xylan conversion in Fig. 5(f). This is because of the presence of high xylose concentrations; xylose is subsequently converted to additional furfural by the only degradation reaction considered 

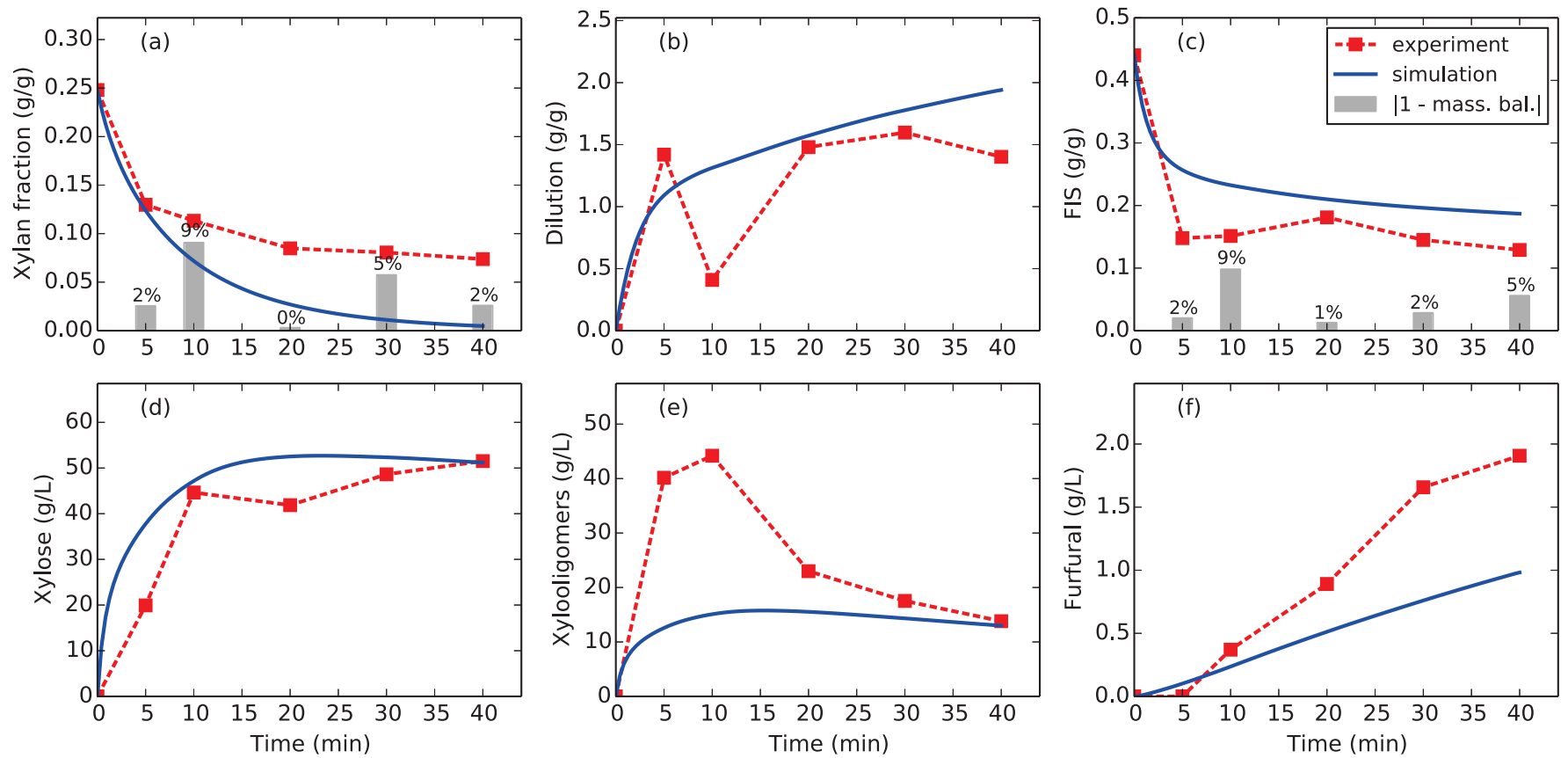

Figure 4: Experimental and simulation results for the same quantities as in Figure 3 for the conditions of $150^{\circ} \mathrm{C}$ steam and $0.5 \%$ acid.

in the model. Other conversion pathways for xylose and furfural to byproducts are known to exist that are not considered in the model. For example, experimental measurements (not shown) suggest that, at severe pretreatment conditions, xylose degradation products may be forming heterogeneous complexes with lignin.

\subsection{Underlying physics}

Figures 7 and 8 show the spatio-temporal variation of different physical and species parameters, respectively, during the initial 30 seconds of pretreatment for the base-case simulations. A "heat-up" (or start-up) time of $\sim 30$ seconds is commonly observed in pretreatment, and it is helpful to have an understanding of the chemical and physical phenomena that are occurring during this initial phase. The temperature distribution within the biomass particle (Fig. $7(\mathrm{c})$ ) tends to equilibrate within 0.1 seconds due to fast thermal diffusion and high lateral heat transfer rate. Figure 7(a) shows the variation 

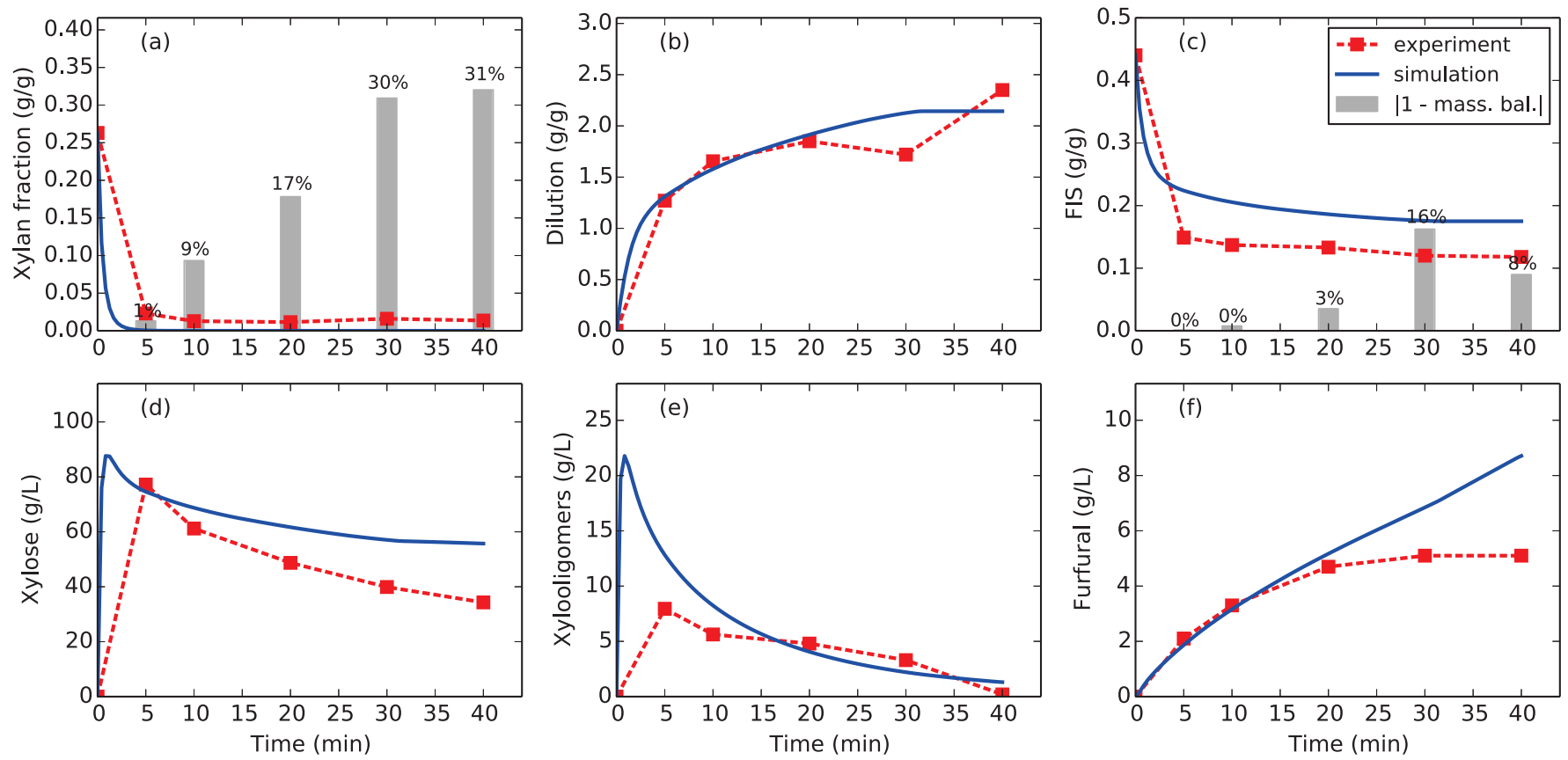

Figure 5: Experimental and simulation results for the same quantities as in Figure 3 for the conditions of $170^{\circ} \mathrm{C}$ steam and $1 \%$ acid.

of steam concentration in the biomass particle. The steam-diffusion coefficient depends on the gas volume fraction, which is the fraction of pores not occupied by liquid. Liquid volume fraction is seen to increase close to the boundaries via condensation (Fig. 7(b)), which reduces the fraction of pores accessible by steam. Therefore, diffusion is impeded at the end of 10 seconds and the steam concentration is seen to stabilize within the biomass particle. The acid concentration is seen to diminish in the regions where liquid fraction increases. Because the acid acts as a catalyst, the total number of moles does not change and acid is diluted at the boundaries where steam condensation first occurs.

Figure 8 shows the spatio-temporal variation of species concentrations during pretreatment. With higher acid concentrations in the middle of the particle, there are higher rates of conversion there as well. Hence, xylan mass fraction is seen to diminish faster in the middle of the particle, and likewise 

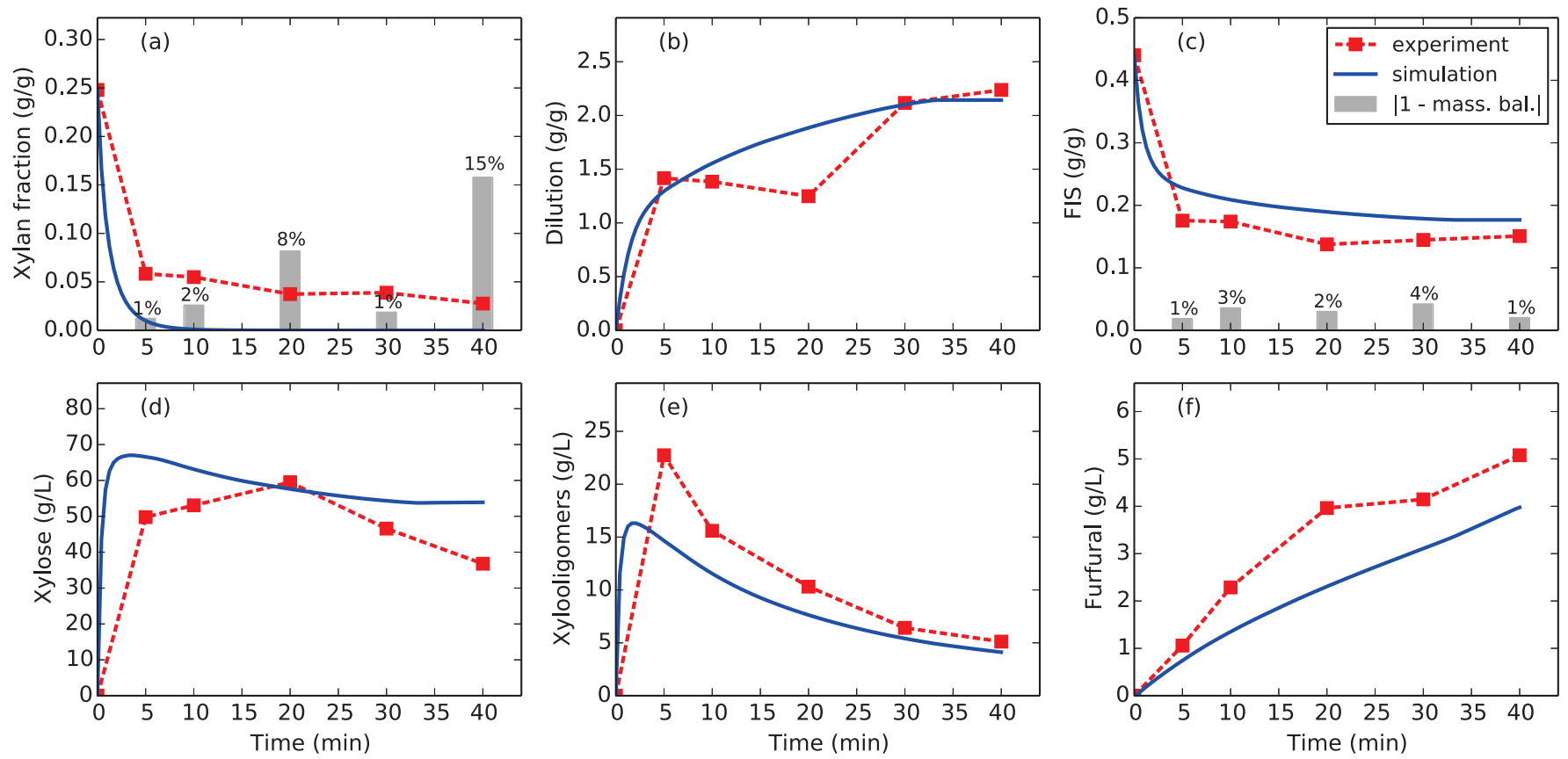

Figure 6: Experimental and simulation results for the same quantities as in Figure 3 for the conditions of $170^{\circ} \mathrm{C}$ steam and $0.5 \%$ acid.

there are higher concentrations of xylose, xylooligomers and furfural (Fig. $8(\mathrm{~b}),(\mathrm{c})$ and $(\mathrm{d}))$ in the middle of the particle.

Figures 9 and 10 show the spatio-temporal variation of pretreatment parameters over longer periods of time, up to $20 \mathrm{~min}$. There is negligible temperature variation within the particle over time, as shown in Fig. 9(c). Steam diffusion is observed to be impeded until 5 minutes and restarts due to reevaporation of liquid at the boundaries. The threshold liquid fraction of $\epsilon_{L T}=0.8$ (see Eq. (13) ) has been reached, which triggers evaporation. The steam diffuses into the particle while the liquid fraction increases via condensation as shown in Fig. 9(b). Acid concentration is seen to reduce in the regions of higher liquid fractions.

Figure 10 shows the spatio-temporal variation of species concentrations during pretreatment. Xylan mass fraction is reduced in the middle of the particle where acid concentration is higher. Higher concentrations of xylose, 

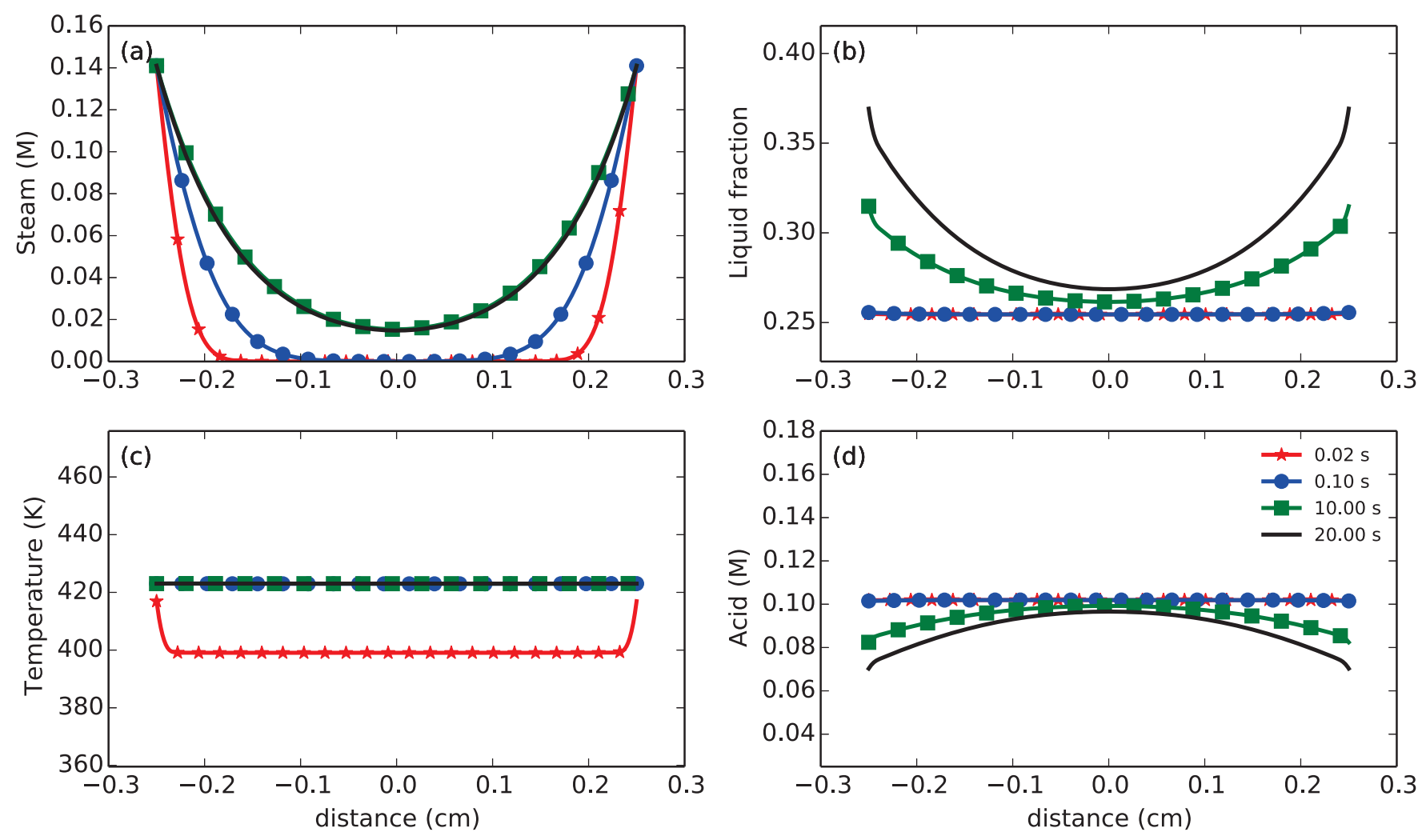

Figure 7: Spatial distribution of (a) steam concentration, (b) liquid volume fraction, (c) temperature, and (d) acid concentration during first 30 seconds of pretreatment at the base-case conditions. 

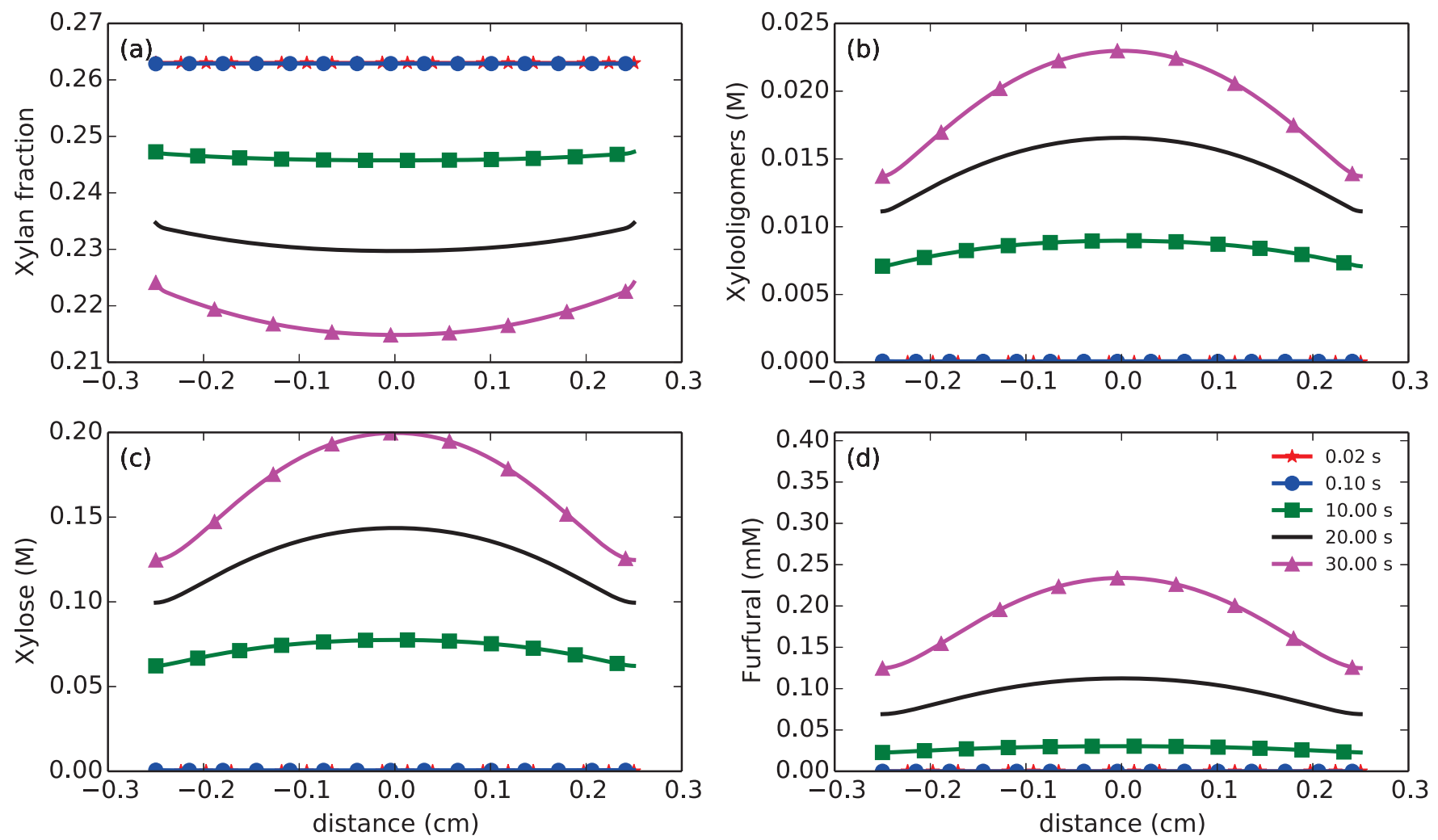

Figure 8: Spatial distribution of (a) xylan mass fraction, (b) xylooligomer concentration, (c) xylose concentration, and (d) furfural concentration during the first 30 seconds of pretreatment at the base-case conditions. 

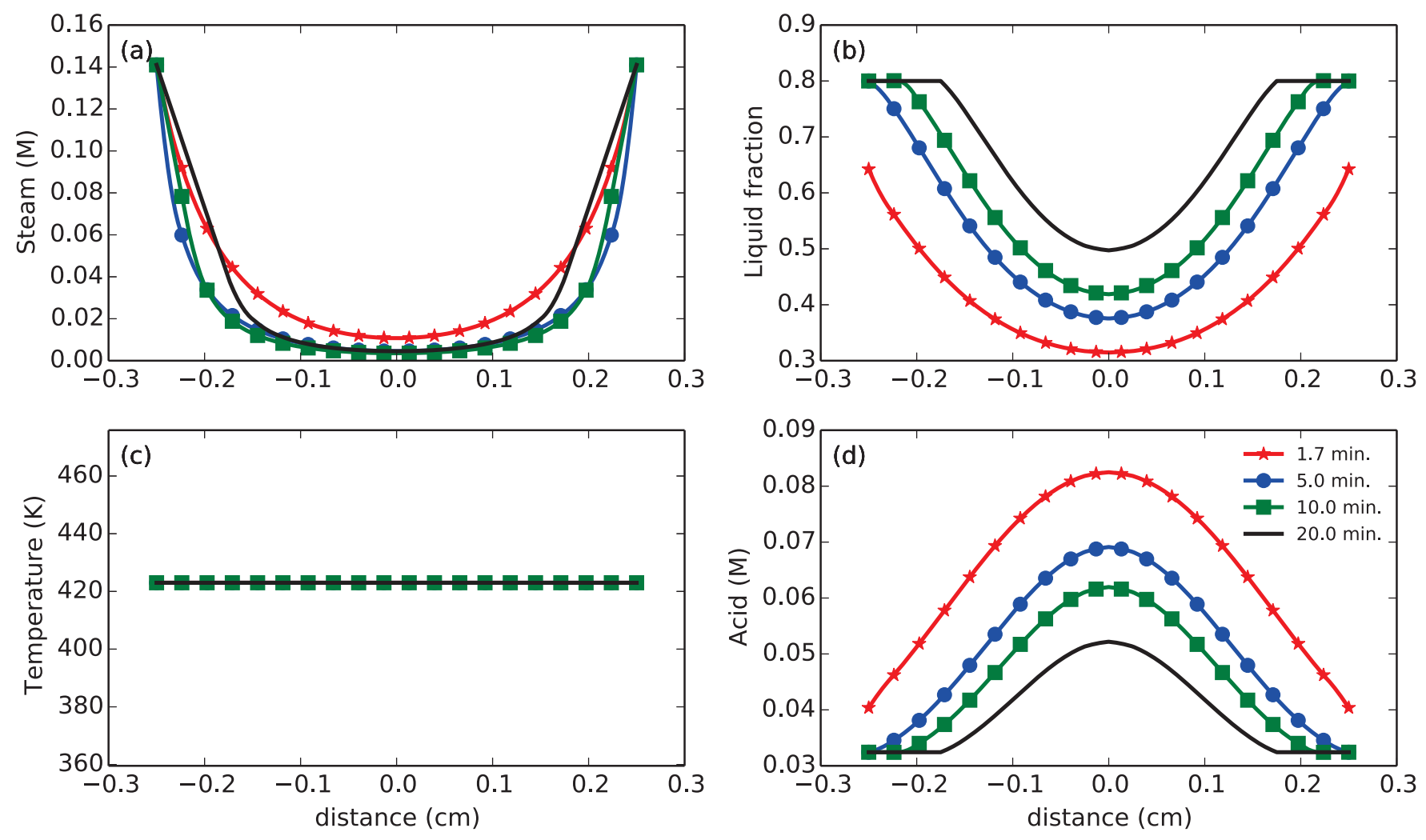

Figure 9: Spatial distribution of (a) steam concentration, (b) liquid volume fraction, (c) temperature, and (d) acid concentration during longer pretreatment times at the base-case conditions. 

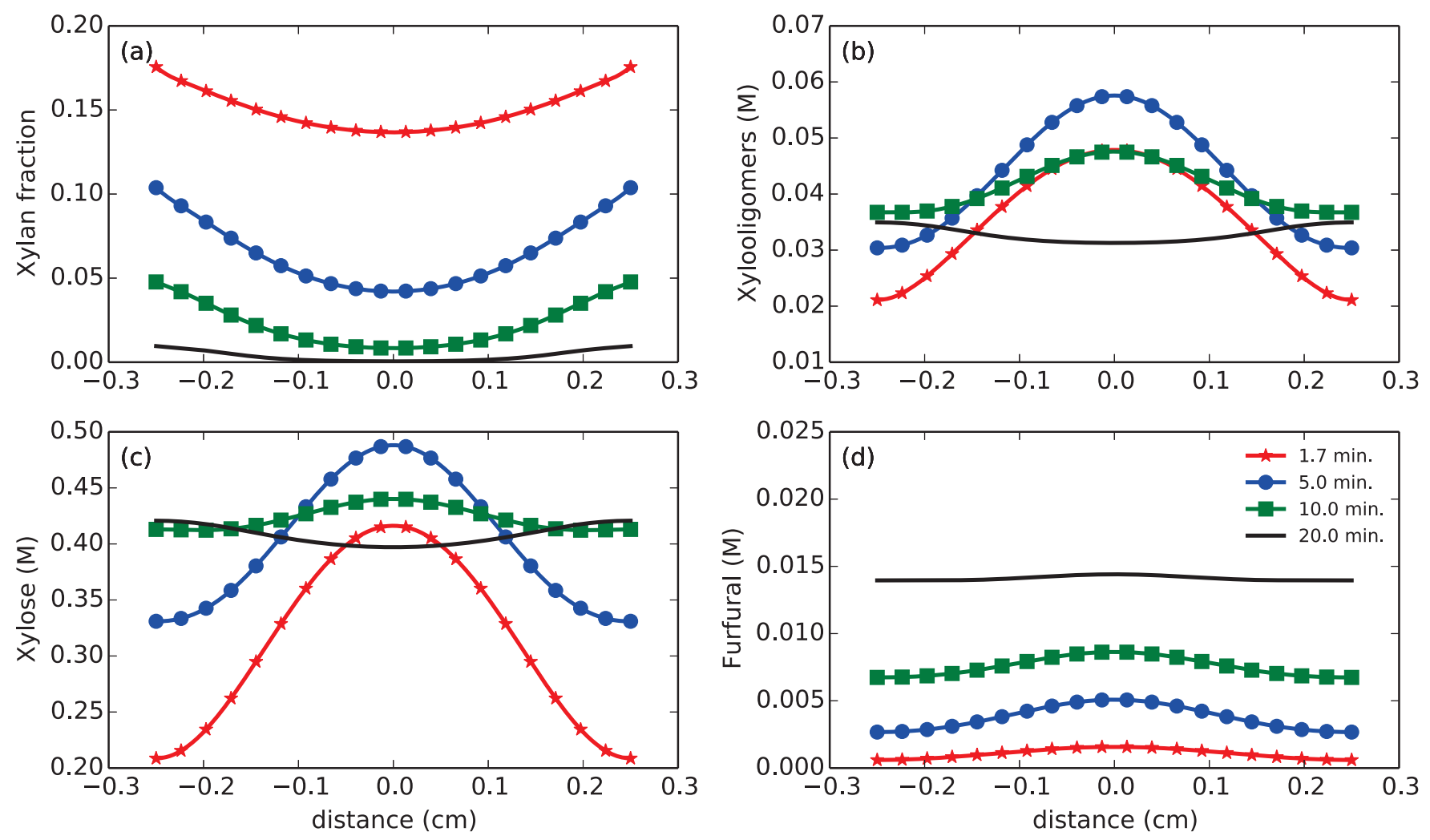

Figure 10: Spatial distribution of (a) xylan mass fraction, (b) xylooligomer concentration, (c) xylose concentration, and (d) furfural concentration during longer pretreatment times at the base-case conditions. 
xylooligomers and furfural are again observed to be in the middle of the particle due to higher acid concentrations that aid in greater species production. Xylan conversion is complete in the middle of the particle at the end of 20 minutes. Oligomer to xylose to furfural conversion becomes the dominant reaction pathway. This along with dilution reduces oligomer and xylose concentrations. Furfural, being the final degradation product, continues to increase in concentration with time as the rate of furfural generation is greater than the rate of steam condensation and subsequent dilution.

\section{Parameter sensitivity study}

A sensitivity study with respect to steam-diffusion coefficient, particle size and aspect ratio is described here. The steam-diffusion coefficient is a function of the pore diameters in the biomass particle as shown in Eq. (9). Pore diameters tend to vary over a wide range of values from nanometers at cell walls to macro-pores, which have sizes on the order of a few microns. Figure 11 shows parameter sensistivities for varying pore diameters where $15 \mathrm{~nm}$ is the base case. The steam-diffusion coefficient at saturation temperature $T_{s}=150^{\circ} \mathrm{C}$ corresponding to the base-case is $0.035 \mathrm{~cm}^{2} / \mathrm{s}$, assuming that the gas volume fraction is equal to one. The Xylan mass fraction is largely insensitive to the diffusion-coefficient variation. The dilution due to condensation is very sensitive to steam diffusion, for which an average variation of $18 \%$ is observed for a pore diameter of $7.5 \mathrm{~nm}$ ( $50 \%$ decrement from base case). The variation in dilution also affects the soluble species concentrations. The variability in dilution reduces to $11 \%$ with a $50 \%$ increase in pore diameter, indicating lower sensitivities for higher steam diffusion rates.

Figure 12 shows the sensitivity of the six different parameters to varying particle lengths. A particle length of $5 \mathrm{~mm}$ was used for the base-case simulations, which is consistent with the knife mill screen size used in the experiments. The amount of dilution is seen to reduce with increasing parti-

cle size. Steam diffusion effects are more prominent in smaller-size particles, 

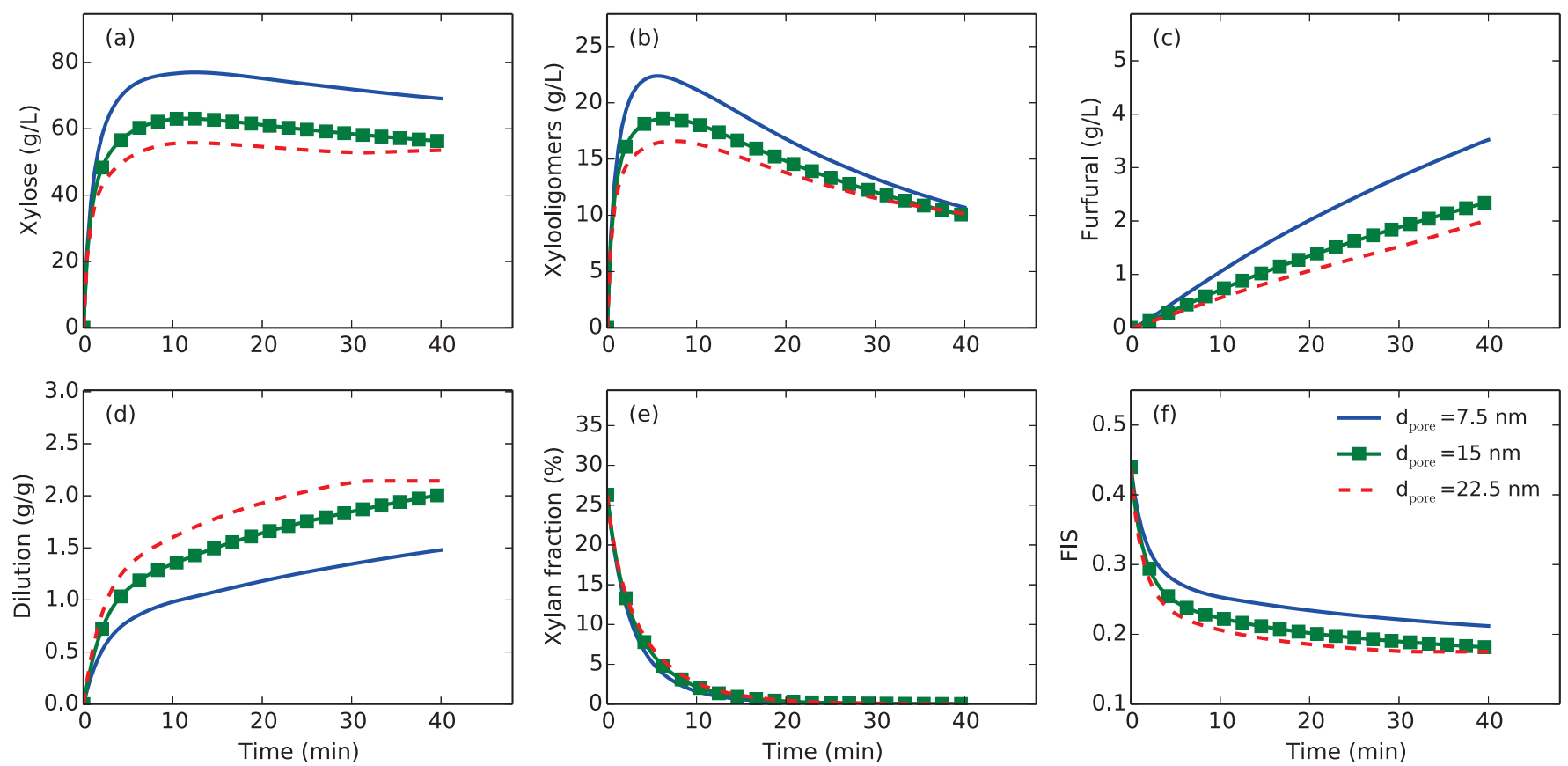

Figure 11: Sensitivity of temporal variation of (a) xylose, (b) xylooligomers, (c) furfural, (d) dilution, (e) xylan mass fraction, and (f) FIS with respect to pore diameters used in the calculation of steam-diffusion coefficient. 

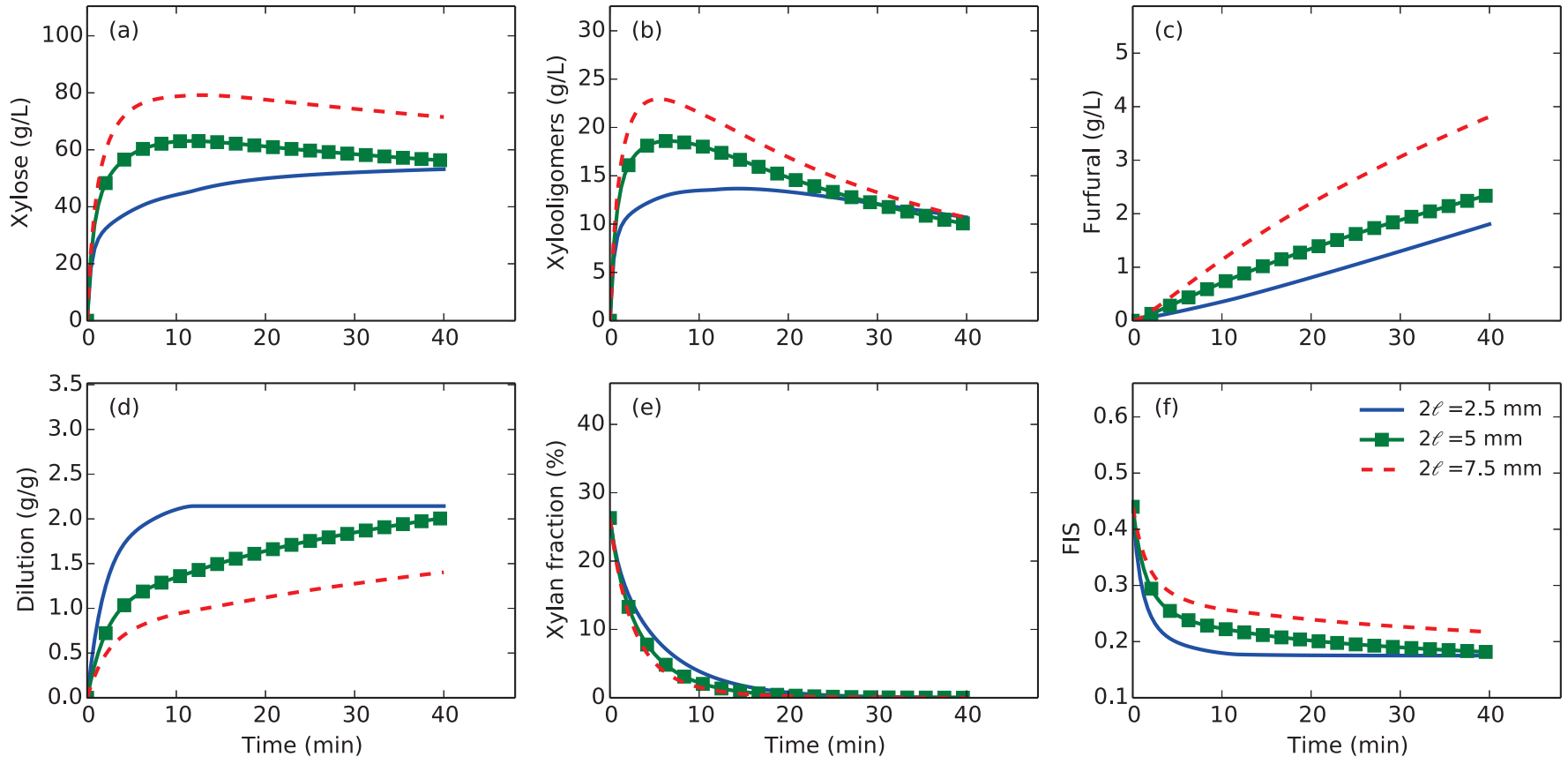

Figure 12: Sensitivity of temporal variation of (a) xylose, (b) xylooligomers, (c) furfural, (d) dilution, (e) xylan mass fraction, and (f) fraction of insoluble solids (FIS) with respect to particle length.

which increases water content over a larger fraction of the particle volume. The sensitivity to dilution is observed in all of the soluble species with higher concentration values for larger particles.

Figure 13 shows the sensitivity of the six different parameters to varying aspect ratios from 15 to 25 . All other variables are set to the same values as the base-case simulations. The results are largely insensitive to aspect ratio. The aspect ratio affects the transverse heat transfer term in the thermal energy equation (Eq. 8). The temperature equilibration happens over seconds, which does not affect species transport for varying aspect ratios.

\section{Conclusions}

A spatio-temporal multiphysics model for a biomass particle undergoing dilute-acid pretreatment at high-solids concentrations has been developed. 

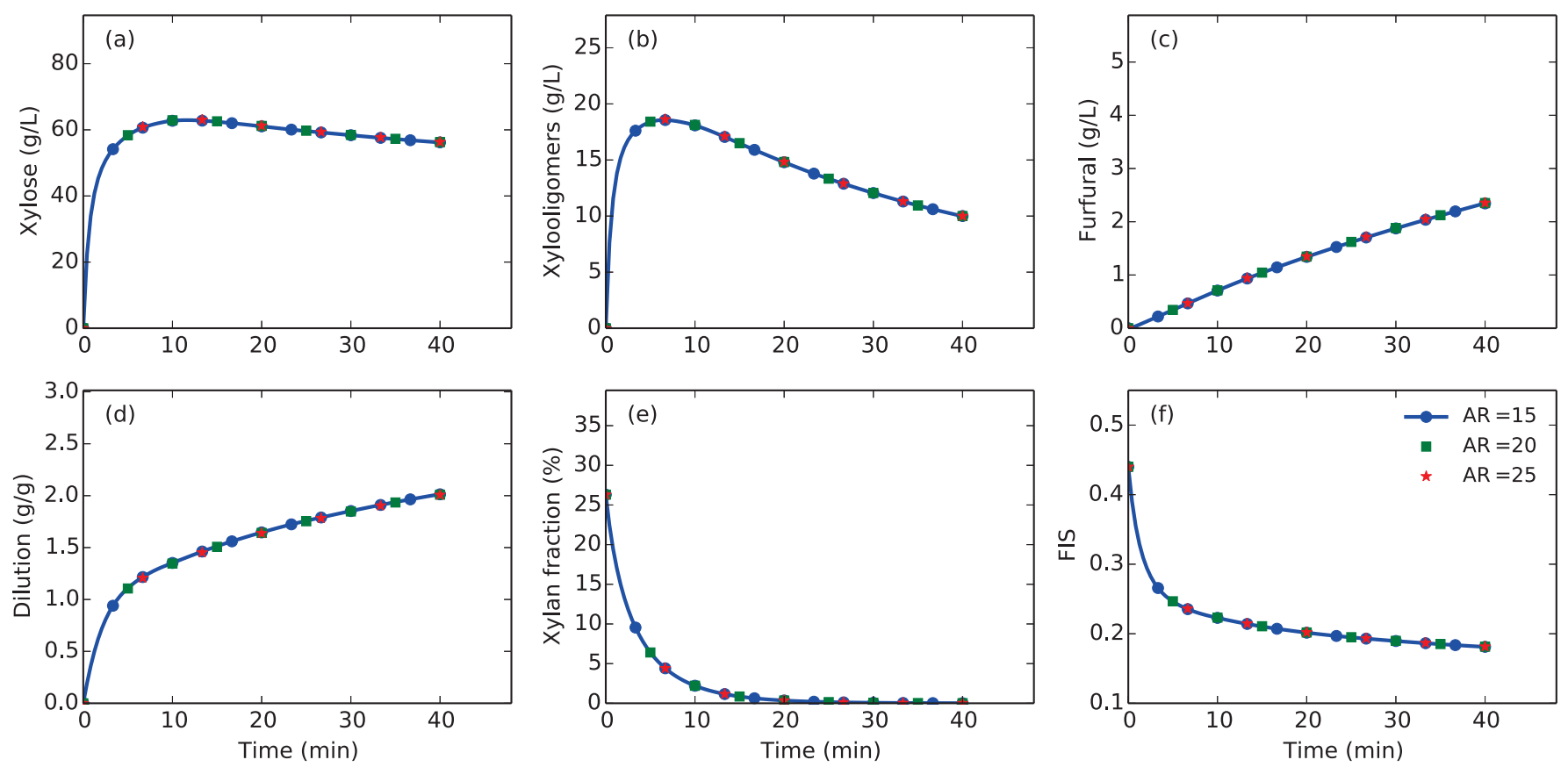

Figure 13: Sensitivity of temporal variation of (a) xylose, (b) xylooligomers, (c) furfural, (d) dilution, (e) xylan mass fraction, and (f) fraction of insoluble solids (FIS) with respect to aspect ratio (AR). 
Model parameters were obtained from the literature, where possible, and by fitting with one set of experiments (base case); the model was then used to predict the results from three other sets of experiments. The model predictions are observed to agree well with the experimental data. Spatio-temporal variation studies illustrate that thermal-conduction time scales are much shorter than reaction time scales, which results in the rapid equilibration of temperature within the biomass particle. Biomass conversion reactions are seen to closely follow spatial and transient variations in acid concentration. Steam diffusion, condensation, and evaporation are competing processes which play an important role in determining the acid concentration in the biomass particle. An intra-particle multiphysics model such as this one could prove to be an effective simulation tool for design and optimization of pretreatment unit operations in bio-chemical conversion processes to biofuels.

\section{Acknowledgments}

The authors would like to thank Dr. Yun Ji (currently at the department of Chemical Engineering, U. North Dakota) for assisting with the pretreatment experiments. This work was funded by the U.S. Department of Energy under Contract No. DE-AC36-08-GO28308 with the National Renewable Energy Laboratory and through the Bioenergy Technologies Office.

\section{References}

[1] V. B. Agbor, N. Cicek, R. Sparling, A. Berlin, D. B. Levin, Biomass pretreatment: Fundamentals toward application, Biotechnol. Adv. 29 (6) (2011) 675-685.

[2] C. Ofori-Boateng, K. Lee, Comparative thermodynamic sustainability assessment of lignocellulosic pretreatment methods for bioethanol production via exergy analysis, Chem. Eng. J. 228 (2013) 162-171. 
[3] M. A. Millett, M. J. Effland, D. F. Caulfield, Influence of fine grinding on the hydrolysis of cellulosic materials-acid vs. enzymatic, Adv. Chem. Ser. 181 (1979) 71-89.

[4] W. R. Grous, A. O. Converse, H. E. Grethlein, Effect of steam explosion pretreatment on pore size and enzymatic hydrolysis of poplar, Enzyme Microb. Technol. 8 (5) (1986) 274-280.

[5] H. K. Murnen, V. Balan, S. P. Chundawat, B. Bals, L. Sousa, B. E. Dale, Optimization of ammonia fiber expansion (AFEX) pretreatment and enzymatic hydrolysis of miscanthus x giganteus to fermentable sugars, Biotechnol. Progr. 23 (4) (2007) 846-850.

[6] A. Esteghlalian, A. G. Hashimoto, J. J. Fenske, M. H. Penner, Modeling and optimization of the dilute-sulfuric-acid pretreatment of corn stover, poplar and switchgrass, Bioresour. Technol. 59 (2) (1997) 129-136.

[7] E. Us, N. Perendeci, Improvement of methane production from greenhouse residues: Optimization of thermal and $\mathrm{H} 2 \mathrm{SO} 4$ pretreatment process by experimental design, Chem. Eng. J. 181 (2012) 120-131.

[8] M. Rocha, T. Rodrigues, T. de Albuquerque, L. Gonçalves, G. de Macedo, Evaluation of dilute acid pretreatment on cashew apple bagasse for ethanol and xylitol production, Chem. Eng. J. 243 (2014) $234-243$

[9] A. B. Bjerre, A. B. Olesen, T. Fernqvist, A. Plöger, A. S. Schmidt, Pretreatment of wheat straw using combined wet oxidation and alkaline hydrolysis resulting in convertible cellulose and hemicellulose, Biotechnol. Bioeng. 49 (5) (1996) 568-577.

[10] E. Gholamzad, K. Karimi, M. Masoomi, Effective conversion of waste polyester-cotton textile to ethanol and recovery of polyester by alkaline pretreatment, Chem. Eng. J. 253 (2014) 40-45. 
[11] H. Tan, K. T. Lee, Understanding the impact of ionic liquid pretreatment on biomass and enzymatic hydrolysis, Chem. Eng. J. 183 (2012) $448-458$.

[12] P. Kumar, D. M. Barrett, M. J. Delwiche, P. Stroeve, Methods for pretreatment of lignocellulosic biomass for efficient hydrolysis and biofuel production, Ind. Eng. Chem. Res. 48 (8) (2009) 3713-3729.

[13] M. P. Tucker, K. H. Kim, M. M. Newman, Q. A. Nguyen, Effects of temperature and moisture on dilute-acid steam explosion pretreatment of corn stover and cellulase enzyme digestibility, Appl. Biochem. Biotechnol. 105 (2003) 165-177.

[14] A. Mittal, S. G. Chatterjee, G. M. Scott, T. E. Amidon, Modeling xylan solubilization during autohydrolysis of sugar maple wood meal: reaction kinetics, Holzforschung 63 (3) (2009) 307-314.

[15] R. R. Gustafson, C. A. Sleicher, W. T. McKean, B. A. Finlayson, Theoretical model of the kraft pulping process, Ind. Eng. Chem. Process Des. Dev. 22 (1) (1983) 87-96.

[16] A. E. Abasaeed, Y. Y. Lee, J. R. Watson, Effect of transient heat transfer and particle size on acid hydrolysis of hardwood cellulose, Bioresour. Technol. 35 (1) (1991) 15-21.

[17] D. Xu, J. Cheng, X. Zhou, A model of heat and moisture transfer through the parallel pore textiles, J. Fiber Bioeng. Inform. 3 (4) (2010) 250-255.

[18] M. A. Sprague, M. E. Colvin, A mixture enthalpy fixed grid model for temperature evolution and heterocyclic amine formation in a frying beef patty, Food Res. Int. 44 (3) (2011) 789-797.

[19] B. H. Davison, J. Parks, M. F. Davis, B. S. Donohoe, Plant cell walls: Basics of structure, chemistry, accessibility and the influence on conver- 
sion, in: C. E. Wyman (Ed.), Aqueous Pretreatment of Plant Biomass for Biological and Chemical Conversion to Fuels and Chemicals, John Wiley \& Sons, 2013, pp. 23-38.

[20] P. N. Ciesielski, W. Wang, X. W. Chen, T. B. Vinzant, M. P. Tucker, S. R. Decker, M. E. Himmel, D. K. Johnson, B. S. Donohoe, Effect of mechanical disruption on the effectiveness of three reactors used for dilute acid pretreatment of corn stover part 2: morphological and structural substrate analysis, Biotechnol. Biofuels 7 (2014) 47. doi:10.1186/17546834-7-47.

[21] J. R. Welty, C. E. Wicks, G. Rorrer, R. E. Wilson, Fundamentals of Momentum, Heat, and Mass Transfer, John Wiley \& Sons, 2009.

[22] C. Chan, N. Zamel, X. Li, J. Shen, Experimental measurement of effective diffusion coefficient of gas diffusion layer/microporous layer in PEM fuel cells, Electrochim. Acta 65 (2012) 13-21.

[23] C. I. Ishizawa, M. F. Davis, D. F. Schell, D. K. Johnson, Porosity and its effect on the digestibility of dilute sulfuric acid pretreated corn stover, J. Agric. Food Chem. 55 (7) (2007) 2575-2581.

[24] R. Karinen, K. Vilonen, M. Niemelä, Biorefining: heterogeneously catalyzed reactions of carbohydrates for the production of furfural and hydroxymethylfurfural, ChemSusChem 4 (8) (2011) 1002-1016.

[25] S. A. Hosseini, N. Shah, Multiscale modelling of biomass pretreatment for biofuels production, Chem. Eng. Res. Des. 87 (9) (2009) 1251-1260.

[26] T. V. Nguyen, R. E. White, A water and heat management model for proton-exchange-membrane fuel cells, J. Electrochem. Soc. 140 (8) (1993) 2178-2186.

[27] E. M. Rønquist, A. T. Patera, A Legendre spectral element method 
for the Stefan problem, Int. J. Numer. Methods Eng. 24 (12) (1987) $2273-2299$.

[28] J. Shekiro, E. M. Kuhn, M. J. Selig, N. J. Nagle, S. R. Decker, R. T. Elander, Enzymatic conversion of xylan residues from dilute acidpretreated corn stover, Appl. Biochem. Biotechnol. 168 (2012) 421-433.

[29] J. B. Sluiter, R. O. Ruiz, C. J. Scarlata, A. D. Sluiter, D. W. Templeton, Compositional analysis of lignocellulosic feedstocks. 1. review and description of methods., J. Agric. Food Chem. 58 (2010) 9043-9053.

[30] D. W. Templeton, C. J. Scarlata, J. B. Sluiter, E. J. Wolfrum, Compositional analysis of lignocellulosic feedstocks. 2. method uncertainties., J. Phys. Chem. 58 (2010) 9054-9062.

[31] C. Karunanithy, Y. Wang, K. Muthukumarappan, S. Pugalendhi, Physiochemical characterization of briquettes made from different feedstocks, Biotechnol. Res. Int. 2012.

[32] W. Simpson, A. Tenwolde, Physical Properties and Moisture Relations of Wood, FPL-GTR-113 Wood Handbook: wood as an Engineering material, USDA Forest Products Laboratory, 1999.

[33] Y. Touloukian, P. Liley, S. Saxena, Thermal conductivity - Nonmetallic liquids and gases, Vol. 3, TPRC Data Series, 1970.

[34] J. Goodykoontz, R. Dorsch, Local Heat-transfer Coefficients for Condensation of Steam in Vertical Downflow Within a 5/8-inch-diameter Tube, NASA, 1966.

[35] N. Kaliyan, Densification of biomass, Ph.D. thesis, University of Minnesota (2008).

[36] Y. S. Touloukian, T. Makita, Specific Heat-Nonmetallic Liquids and Gases, Vol. 6S, TPRC Data Series, 1976. 
[37] A. Saxena, A. Ragauskas, Water transmission barrier properties of biodegradable films based on cellulosic whiskers and xylan, Carbohydr. Polym. 78 (2) (2009) 357-360.

[38] J. Brandrup, E. Immergut, E. Grulke, A. Abe, D. Bloch, Polymer Handbook, Vol. 89, Wiley New York, 1999.

[39] T. Hu, Chemical modification, properties and usage of Lignin, Springer, 2002.

[40] R. Kankanamge, Xylan depolymerisation under subcritical water conditions, Ph.D. thesis, University of Sydney (2013). 